The Number of Homomor phi sms from a Fi ni te Abel i an Group to a Symmet ri c Group (II)

\begin{tabular}{|l|l|}
\hline 著者 & TAKEGAHARA Yugen \\
\hline $\begin{array}{l}\text { j our nal or } \\
\text { publ i cat } \mathrm{i} \text { on } \mathrm{t} \text { i t l e }\end{array}$ & Communi cat $\mathrm{i}$ ons i $\mathrm{n}$ Al gebr a \\
\hline vol une & 44 \\
\hline number & 6 \\
\hline page $\mathrm{r}$ ange & $2402-2442$ \\
\hline year & $2016-04-29$ \\
\hline URL & ht t p: //hdl . handl e. net /10258/00008937 \\
\hline
\end{tabular}




\title{
THE NUMBER OF HOMOMORPHISMS FROM A FINITE ABELIAN GROUP TO A SYMMETRIC GROUP (II)
}

\author{
Yugen Takegahara \\ Muroran Institute of Technology, 27-1 Mizumoto, \\ Muroran 050-8585, Japan \\ E-mail: yugen@mmm.muroran-it.ac.jp
}

\begin{abstract}
For any finite abelian group $A$, we give the lower bound of $\operatorname{ord}_{p}\left(\left|\operatorname{Hom}\left(A, S_{n}\right)\right|\right)$, and determine the region of convergence of the $p$-adic power series $1+\sum_{n=1}^{\infty}\left|\operatorname{Hom}\left(A, S_{n}\right)\right| X^{n} / n$ !.
\end{abstract}

\section{Introduction}

Let $A$ be a finite abelian group and $h_{n}(A)$ the number of homomorphisms from $A$ to the symmetric group $S_{n}$ on $n$ letters. For convenience, we put $h_{0}(A)=1$. We denote by $E_{A}(X)$ the exponential generating function of the sequence $\left\{h_{n}(A)\right\}$, i.e., $E_{A}(X)=\sum_{n=0}^{\infty} h_{n}(A) X^{n} / n$ !. According to [20],

$$
E_{A}(X)=\exp \left(\sum_{d=1}^{\infty} \frac{m_{A}(d)}{d} X^{d}\right)
$$

where $m_{A}(d)$ denotes the number of subgroups of index $d$ in $A$.

Let $p$ be a prime. It follows from $[17,21]$ that $h_{n}(A)$ is a multiple of $\operatorname{gcd}(|A|, n !)$. This property interests us in $p$-divisibility of $h_{n}(A)$. Using the generating function above we research into $\operatorname{ord}_{p}\left(h_{n}(A)\right)$. Here $\operatorname{ord}_{p}(a)$ denotes 2000 Mathematics Subject Classification: Primary 05A15; Secondary 20B30; 20K01; 20K27. Keyword : group homomorphism; symmetric group; p-adic exponential function. 
the exponent of $p$ in the decomposition of $a$ into prime factors for each non-zero integer $a$. For the notation and terminology, see [10].

We denote by $C_{p^{u}}$ the finite cyclic group of order $p^{u}$. Since $h_{n}\left(C_{p}\right)$ is a multiple of $\operatorname{gcd}(p, n !)$, it follows that $\operatorname{ord}_{p}\left(h_{n}\left(C_{p}\right)\right) \geq 1$ for $n \geq p$; however, this inequality does not indicate the real value of $\operatorname{ord}_{p}\left(h_{n}\left(C_{p}\right)\right)$. If a Sylow $p$-subgroup of $A$ is the direct product of two cyclic $p$-groups, the properties of $\operatorname{ord}_{p}\left(h_{n}(A)\right)$ are available in $[9,18]$. To take an example, $\operatorname{ord}_{p}\left(h_{n}\left(C_{p}\right)\right) \geq$ $[n / p]-\left[n / p^{2}\right]$ (see also $\left.[4,5,6,7]\right)$. Here $[x]$ denotes the largest integer not exceeding $x$ for each real number $x$. If $p=2$, this assertion is equivalent to [3, Theorem 10].

In this paper, we generalize the results shown in [9]. Especially, we know the best lower bound of $\operatorname{ord}_{p}\left(h_{n}(A)\right)$ (cf. Theorem 1.1) so that we can get the region of convergence of the $p$-adic power series $E_{A}(X)$ (cf. Corollary 1.1).

Let $s$ be a nonnegative integer. For nonnegative integers $\lambda_{1}, \lambda_{2}, \ldots$ such that $\lambda_{1} \geq \lambda_{2} \geq \cdots$ and $s=\lambda_{1}+\lambda_{2}+\cdots$, the sequence $\lambda=\left(\lambda_{1}, \lambda_{2}, \ldots\right)$ is called a partition of $s$. Any partition of $s$ contains only finitely many nonzero terms. If $\lambda=\left(\lambda_{1}, \lambda_{2}, \ldots\right)$ is a partition of $s$ and if a finite abelian $p$-group $P$ is isomorphic to the direct product of cyclic $p$-groups $C_{p^{\lambda_{i}}}, i=1,2, \ldots$, i.e., $P \simeq C_{p^{\lambda_{1}}} \times C_{p^{\lambda_{2}}} \times \cdots$, then $\lambda$ is called the type of $P$.

Let $\alpha_{\lambda}(i ; p)$ denote the number of subgroups of order $p^{i}$ in a finite abelian $p$-group of type $\lambda$. It is well known that $\alpha_{\lambda}(i ; p)$ is a polynomial in $p$ with nonnegative integer coefficients, which depends only on $\lambda$ and $i$. In order to study $E_{A}(X)$, we need the properties of $\alpha_{\lambda}(i ; p)$ shown in $[1,15]$. For instance, we use Butler's unimodality result [1, Theorem], namely,

For a partition $\lambda$ of $s$, if $i$ is a positive integer less than or equal to [s/2], then $\alpha_{\lambda}(i ; p)-\alpha_{\lambda}(i-1 ; p)$ has nonnegative coefficients.

Throughout the paper, $\lambda=\left(\lambda_{1}, \lambda_{2}, \ldots\right)$ denotes a partition of $s$. Let

$$
u=\max \left\{\lambda_{1},\left[\frac{s+1}{2}\right]\right\}
$$

and $v=s-u=\min \left\{s-\lambda_{1},[s / 2]\right\}$. For brevity's sake, we define

$$
f_{p}^{k}(n):= \begin{cases}\sum_{j=1}^{k}\left[\frac{n}{p^{j}}\right] & \text { if } k \text { is a positive integer, } \\ 0 & \text { if } k \text { is a nonpositive integer }\end{cases}
$$


and

$$
\tau_{\lambda}^{p}(n):= \begin{cases}f_{2}^{u}(n)+\left[\frac{n}{2^{u+2}}\right]-\left[\frac{n}{2^{u+3}}\right] & \text { if } p=2 \text { and if } 2 u=s \geq 2, \\ f_{p}^{u}(n)-(2 u-s)\left[\frac{n}{p^{u+1}}\right] & \text { otherwise. }\end{cases}
$$

Our goal in this paper is to establish the following theorem (see Section 8).

Theorem 1.1 Let $A$ be a finite abelian group, and suppose that $A$ possesses a Sylow p-subgroup of type $\lambda$.

(1) We have $\operatorname{ord}_{p}\left(h_{n}(A)\right) \geq \tau_{\lambda}^{p}(n)$. Excepting the case where $p=2$ and $2 u=s \geq 2$, if $n$ is a multiple of $p^{u+1}$, then $\operatorname{ord}_{p}\left(h_{n}(A)\right)=\tau_{\lambda}^{p}(n)$.

(2) Suppose that $p=2$ and that $2 u=s \geq 2$. If $n$ is a multiple of $2^{u+3}$, then $\operatorname{ord}_{2}\left(h_{n}(A)\right)=\tau_{\lambda}^{2}(n)$.

For part (2) of Theorem 1.1, a detailed result is seen in Corollary 8.2. When $2 u=s \geq 2$, the difference between the cases where $p>2$ and $p=2$ comes from that between Lemmas 2.4 and 2.6 which are the keys to proving Theorem 1.1 with $A=C_{p^{u}} \times C_{p^{v}}$ (see Theorems 2.1 and 2.2).

The radius of convergence of the $p$-adic power series $E_{A}(X)$ is $p^{a}$ where

$$
a=\lim _{n \rightarrow \infty} \inf \frac{\operatorname{ord}_{p}\left(h_{n}(A)\right)-\operatorname{ord}_{p}(n !)}{n},
$$

so that we can get the following corollary to Theorem 1.1 (see Section 9).

Corollary 1.1 Under the hypothesis of Theorem 1.1, the p-adic power series $E_{A}(X)$ converges only in the open disc of radius $p^{a}$ where

$$
a= \begin{cases}-\frac{7}{2^{u+3}} & \text { if } p=2 \text { and if } 2 u=s \geq 2, \\ -\frac{1}{p^{u}(p-1)}-\frac{2 u-s}{p^{u+1}} & \text { otherwise. }\end{cases}
$$

Many results in this paper are based on the results in [9]. In Section 2 we give an alternative proof of [9, Theorem 3.1] and that of [9, Theorem 4.2] except the second assertion. The second assertion of [9, Theorem 4.2] with $l \geq 2$ is wrong; but [9, Theorem 4.1], which states the first two assertions of [9, Theorem 4.2] with $l=1$, is true nevertheless. In Section 7 we correct an error in [9, Theorem 4.2] (see Theorem 7.1). 
We state a brief outline of the proof of Theorem 1.1. Sections 3 and 4 are devoted to analyses of $\alpha_{\lambda}(i ; p)$ and $E_{P}(X)$ for a finite abelian $p$-group $P$. The first statement of (1) with $A=P$ is proved in Section 5 . We continue to discuss the properties of $h_{n}(P)$ in Sections 6 and 7. The theorems on $h_{n}(P)$ are extended to those on $h_{n}(A)$, and consequently, Theorem 1.1 is proved at the end of Section 8.

\section{The direct product of two cyclic $p$-groups}

In this section we assume that $\lambda=(u, v, 0, \ldots)$ and that $P$ is the direct product of two cyclic $p$-groups $C_{p^{u}}$ and $C_{p^{v}}$. Here $u \geq v \geq 0$.

Let $H$ be a finite group, and let $H^{(n)}$ be the direct product of $n$ copies of $H$. Let $H \backslash S_{n}$ be the wreath product of $H$ with $S_{n}$. Hence every element of $H \prec S_{n}$ is written as $\left(h_{1}, h_{2}, \ldots, h_{n}\right) \pi$ for $\left(h_{1}, h_{2}, \ldots, h_{n}\right) \in H^{(n)}$ and $\pi \in S_{n}$, and the product of two elements of $H<S_{n}$ is defined by

$$
\left(h_{1}, h_{2}, \ldots, h_{n}\right) \pi \cdot\left(h_{1}^{\prime}, h_{2}^{\prime}, \ldots, h_{n}^{\prime}\right) \pi^{\prime}=\left(h_{1} h_{\pi^{-1}(1)}^{\prime}, h_{2} h_{\pi^{-1}(2)}^{\prime}, \ldots, h_{n} h_{\pi^{-1}(n)}^{\prime}\right) \pi \pi^{\prime}
$$

for all $\left(h_{1}, h_{2}, \ldots, h_{n}\right) \pi,\left(h_{1}^{\prime}, h_{2}^{\prime}, \ldots, h_{n}^{\prime}\right) \pi^{\prime} \in H \curlyvee S_{n}$.

Let $d$ be a positive integer, and let $C_{d}$ be a finite cyclic group of order $d$. We define $h\left(C_{d}, H\right):=\sharp\left\{x \in H \mid x^{d}=1\right\}$. So $h\left(C_{d}, H\right)$ is just the number of homomorphisms from $C_{d}$ to $H$.

Put $h_{n}\left(C_{d} ; H\right)=h\left(C_{d}, H<S_{n}\right)$ if $n \geq 1$, and $h_{0}\left(C_{d} ; H\right)=1$.

The centralizer of a permutation that factorizes $i$ disjoint cycles of length $p^{u+1}$ in $S_{n}$, where $0 \leq i \leq\left[n / p^{u+1}\right]$, is isomorphic to the direct product $\left(C_{p^{u+1}} \prec S_{i}\right) \times S_{n-p^{u+1} i}$ of $C_{p^{u+1}} \prec S_{i}$ and $S_{n-p^{u+1} i}$, and

$$
\left|\left(C_{p^{u+1}} \prec S_{i}\right) \times S_{n-p^{u+1} i}\right|=p^{(u+1) i} i !\left(n-p^{u+1} i\right) !
$$

(see $[8,4.1 .19])$. Hence the following lemma, which is $[18,(10)]$, holds.

Lemma 2.1 Let $y_{n}=\left[n / p^{u+1}\right]$ for each nonnegative integer $n$. Then

$$
h_{n}\left(C_{p^{u+1}} \times C_{p^{v}}\right)=\sum_{i=0}^{y_{n}} \frac{n ! h_{i}\left(C_{p^{v}} ; C_{p^{u+1}}\right)}{p^{(u+1) i} i !\left(n-p^{u+1} i\right) !} h_{n-p^{u+1} i}(P) .
$$

We use the fact that $\operatorname{ord}_{p}(n !)=\sum_{j=1}^{\infty}\left[n / p^{j}\right]([14$, p. 242]), and also use $[9$, Lemma 2.1], namely, 
Lemma 2.2 For each integer $i$ with $0 \leq i \leq\left[n / p^{u+1}\right]$,

$$
\begin{aligned}
\operatorname{ord}_{p}\left(\frac{n !}{i !\left(n-p^{u+1} i\right) !}\right)=f_{p}^{u+1} & \left(p^{u+1} i\right) \\
& +\sum_{j=u+2}^{\infty}\left(\left[\frac{n}{p^{j}}\right]-\left[\frac{p^{u+1} i}{p^{j}}\right]-\left[\frac{n-p^{u+1} i}{p^{j}}\right]\right) .
\end{aligned}
$$

Let $\left\{d_{0}, d_{1}, \ldots\right\}$ be the set consisting of all divisors of $d$. Let $r=d_{k}$ for some $k$, and suppose that $\pi^{\prime}=\left(i_{1} i_{2} \cdots i_{r}\right)$ is a cycle of length $r$ in $S_{n}$. Then the number of elements $\left(h_{1}, h_{2}, \ldots, h_{n}\right) \pi^{\prime}$ in $H<S_{n}$ such that

$$
\left(\left(h_{1}, h_{2}, \ldots, h_{n}\right) \pi^{\prime}\right)^{d}=e
$$

and $h_{i}=e_{H}$ for any $i$ with $i \neq i_{1}, i_{2}, \ldots, i_{r}$ is $|H|^{r-1} h\left(C_{d / r}, H\right)$. Here $e$ and $e_{H}$ are the identities of $H \backslash S_{n}$ and $H$, respectively. Hence, if $\pi$ is a permutation in $S_{n}$ that factorizes disjoint $j_{k}$ cycles of length $d_{k}$ for $k=0,1,2, \ldots$, then the number of elements $\left(h_{1}, h_{2}, \ldots, h_{n}\right) \zeta$ in $H<S_{n}$ such that

$$
\left(\left(h_{1}, h_{2}, \ldots, h_{n}\right) \zeta\right)^{d}=e
$$

and $\zeta$ is conjugate to $\pi$ in $S_{n}$ is

$$
\frac{n !}{\prod_{k \geq 0} d_{k}^{j_{k}} j_{k} !} \prod_{k \geq 0}|H|^{\left(d_{k}-1\right) j_{k}} h\left(C_{d / d_{k}}, H\right)^{j_{k}}
$$

(see also $[8,4.2])$. This means that

$$
h_{n}\left(C_{d} ; H\right)=\sum_{j_{0} d_{0}+j_{1} d_{1}+\cdots=n} \frac{n !}{\prod_{k \geq 0} d_{k}^{j_{k}} j_{k} !} \prod_{k \geq 0}|H|^{\left(d_{k}-1\right) j_{k}} h\left(C_{d / d_{k}}, H\right)^{j_{k}},
$$

where the summation runs over all sequences $\left(j_{0}, j_{1}, \ldots\right)$ of nonnegative integers $j_{0}, j_{1}, \ldots$ with $j_{0} d_{0}+j_{1} d_{1}+\cdots=n$.

Lemma 2.3 Suppose that $e$ and $m$ are integers with $e \geq m \geq 0$. Then

$$
h_{n}\left(C_{p^{m}} ; C_{p^{e}}\right)=\sum_{j_{0}+j_{1} p+\cdots+j_{m} p^{m}=n} c_{j_{0}, \ldots, j_{m}} p^{m n+\sum_{k=0}^{m} r_{p}(k) j_{k}},
$$

where $r_{p}(k)=(e-m)\left(p^{k}-1\right)-k$ and

$$
c_{j_{0}, \ldots, j_{m}}=\frac{n !}{\prod_{k=0}^{m} p^{k j_{k}} j_{k} !} \in \mathbb{Z} .
$$


Proof. By (A), we have

$$
h_{n}\left(C_{p^{m}} ; C_{p^{e}}\right)=\sum_{j_{0}+j_{1} p+\cdots+j_{m} p^{m}=n} \frac{n !}{\prod_{k \geq 0} p^{k j_{k}} j_{k} !} \prod_{k=0}^{m} p^{e\left(p^{k}-1\right) j_{k}+(m-k) j_{k}} .
$$

The lemma is an immediate consequence of this fact.

The next lemma is stated in [18, Lemma 2.5].

Lemma 2.4 Suppose that $e$ and $m$ are integers with $e>m \geq 0$. Then

$$
h_{n}\left(C_{p^{m}} ; C_{p^{e}}\right) \equiv p^{m n} \bmod p^{m n+e-m-1+\delta},
$$

where $\delta=1$ if either $p>2$ or $m=0$, and $\delta=0$ otherwise.

Proof. If $m=0$, then the assertion clearly holds. Assume that $m \geq 1$. For each integer $k$ with $1 \leq k \leq m$,

$$
\begin{aligned}
r_{p}(k)=(e-m)\left(p^{k}-1\right)-k & =(e-m-1)\left(p^{k}-1\right)+p^{k}-(k+1) \\
& \geq e-m-1+\delta,
\end{aligned}
$$

and thereby, Lemma 2.3 deduces the assertion.

We give an alternative proof, which is sketched in [18], of [9, Theorem 3.1], namely,

Theorem 2.1 Let $y_{n}=\left[n / p^{u+1}\right]$ for each nonnegative integer $n$. Then

$$
\operatorname{ord}_{p}\left(h_{n}(P)\right) \geq f_{p}^{u}(n)-(u-v) y_{n}
$$

and

$$
h_{n}(P) \equiv \frac{(-1)^{y_{n}} n !}{p^{(u-v+1) y_{n}} y_{n} !\left(n-p^{u+1} y_{n}\right) !} h_{n-p^{u+1} y_{n}}(P) \quad \bmod p^{f_{p}^{u}(n)-(u-v)\left(y_{n}-1\right)+\delta},
$$

where $\delta=1$ if either $p>2$ or $v=0$, and $\delta=0$ otherwise.

Proof. We use induction on $y_{n}$. Suppose that $y_{n}=0$. Then $n<p^{u+1}$, and hence $h_{n}\left(C_{p^{u+k}} \times C_{p^{v}}\right)=h_{n}(P)$ for any nonnegative integer $k$. This, combined with [21, Main Theorem] (see also [17]), shows that

$$
\operatorname{ord}_{p}\left(h_{n}(P)\right) \equiv 0 \quad \bmod \operatorname{gcd}\left(p^{u+k}, n !\right)
$$


for any nonnegative integer $k$. Thus $\operatorname{ord}_{p}\left(h_{n}(P)\right) \geq \operatorname{ord}_{p}(n !)=f_{p}^{u}(n)$. Moreover, the second assertion clearly holds. We next suppose that $y_{n} \geq 1$. By the inductive assumption,

$$
\operatorname{ord}_{p}\left(h_{n}\left(C_{p^{u+1}} \times C_{p^{v}}\right)\right) \geq f_{p}^{u+1}(n)-(u+1-v)\left[\frac{n}{p^{u+2}}\right] .
$$

Furthermore,

$$
\begin{aligned}
f_{p}^{u+1}(n)-(u+1 & -v)\left[\frac{n}{p^{u+2}}\right] \\
& =f_{p}^{u}(n)-(u-v) y_{n}+(u+1-v)\left(y_{n}-\left[\frac{y_{n}}{p}\right]\right) \\
& \geq f_{p}^{u}(n)-(u-v) y_{n}+u-v+1,
\end{aligned}
$$

and thereby,

$$
\operatorname{ord}_{p}\left(h_{n}\left(C_{p^{u+1}} \times C_{p^{v}}\right)\right) \geq f_{p}^{u}(n)-(u-v)\left(y_{n}-1\right)+1 .
$$

Hence we can use Lemma 2.1 to get

$$
\sum_{i=0}^{y_{n}} \frac{n ! h_{i}\left(C_{p^{v}} ; C_{p^{u+1}}\right)}{p^{(u+1) i} i !\left(n-p^{u+1} i\right) !} h_{n-p^{u+1} i}(P) \equiv 0 \quad \bmod p^{f_{p}^{u}(n)-(u-v)\left(y_{n}-1\right)+1} .
$$

By Lemma 2.2 and the inductive assumption,

$$
\begin{aligned}
& \operatorname{ord}_{p}\left(\frac{n !}{p^{(u+1) i} i !\left(n-p^{u+1} i\right) !} h_{n-p^{u+1} i}(P)\right) \\
& \quad \geq f_{p}^{u}\left(p^{u+1} i\right)-u i+f_{p}^{u}\left(n-p^{u+1} i\right)-(u-v)\left[\frac{n-p^{u+1} i}{p^{u+1}}\right] \\
& \quad=f_{p}^{u}(n)-(u-v) y_{n}-v i
\end{aligned}
$$

for any integer $i$ with $1 \leq i \leq y_{n}$. The first assertion now follows from Lemma 2.4 and $(\mathrm{C})$. Furthermore, by the inductive assumption,

$$
\begin{aligned}
h_{n}(P) & \equiv-\sum_{i=1}^{y_{n}} \frac{n ! h_{i}\left(C_{p^{v}} ; C_{p^{u+1}}\right)}{p^{(u+1) i} i !} \cdot \frac{h_{n-p^{u+1} i}(P)}{\left(n-p^{u+1} i\right) !} \\
& \equiv-\sum_{i=1}^{y_{n}} \frac{n !}{p^{(u-v+1) i} i !} \cdot \frac{(-1)^{y_{n}-i}}{p^{(u-v+1)\left(y_{n}-i\right)}\left(y_{n}-i\right) !} \cdot \frac{h_{n-p^{u+1} y_{n}}(P)}{\left(n-p^{u+1} y_{n}\right) !} \\
& \equiv-\left\{\sum_{i=1}^{y_{n}} \frac{(-1)^{i} y_{n} !}{i !\left(y_{n}-i\right) !}\right\} \frac{(-1)^{y_{n}} n !}{p^{(u-v+1) y_{n}} y_{n} !\left(n-p^{u+1} y_{n}\right) !} h_{n-p^{u+1} y_{n}}(P) \\
& \equiv \frac{(-1)^{y_{n}} n !}{p^{(u-v+1) y_{n}} y_{n}\left(n-p^{u+1} y_{n}\right) !} h_{n-p^{u+1} y_{n}}(P) \bmod p^{f_{p}^{u}(n)-(u-v)\left(y_{n}-1\right)+\delta},
\end{aligned}
$$


completing the proof.

We turn to the case where $p=2$ and $u=v \geq 1$. By (B),

$$
\sum_{n=0}^{\infty} \frac{h_{n}\left(C_{2} ; C_{2}\right)}{n !} X^{n}=\sum_{n=0}^{\infty}\left(\sum_{j_{0}+2 j_{1}=n} \frac{2^{j_{0}}}{j_{0} ! j_{1} !}\right) X^{n}=\exp \left(2 X+X^{2}\right)
$$

(see also [13, Proposition 3.4]), whence

$$
\sum_{n=0}^{\infty} \frac{h_{n}\left(C_{2} ; C_{2}\right)}{n !} X^{n}=\exp \left(X+\frac{X^{2}}{2}\right)^{2}=\left(\sum_{n=0}^{\infty} \frac{h_{n}\left(C_{2}\right)}{n !} X^{n}\right)^{2} .
$$

The following lemma is due to Tomoyuki Yoshida.

Lemma 2.5 We have

$$
\operatorname{ord}_{2}\left(h_{n}\left(C_{2} ; C_{2}\right)\right)=\left[\frac{n+1}{2}\right] .
$$

Proof. By (D), we have

$$
\sum_{n=1}^{\infty} \frac{h_{n}\left(C_{2} ; C_{2}\right)}{(n-1) !} X^{n-1}=\sum_{n=0}^{\infty} \frac{h_{n}\left(C_{2} ; C_{2}\right)}{n !} 2 X^{n}+\sum_{n=0}^{\infty} \frac{h_{n}\left(C_{2} ; C_{2}\right)}{n !} 2 X^{n+1} .
$$

Hence, if $n \geq 2$, then

$$
h_{n}\left(C_{2} ; C_{2}\right)=2 h_{n-1}\left(C_{2} ; C_{2}\right)+2(n-1) h_{n-2}\left(C_{2} ; C_{2}\right)
$$

([2]).We use induction on $n$. If $n \leq 1$, then the assertion clearly holds. Suppose that $n \geq 2$. By the inductive assumption, $\operatorname{ord}_{2}\left(h_{m}\left(C_{2} ; C_{2}\right)\right)=[(m+1) / 2]$ for any nonnegative integer $m$ less than $n$. Hence we have

$$
\operatorname{ord}_{2}\left(h_{n}\left(C_{2} ; C_{2}\right)\right)=1+\min \left\{\left[\frac{n}{2}\right],\left[\frac{n-1}{2}\right]+\alpha\right\}
$$

where

$$
\alpha=\left\{\begin{array}{cl}
0 & \text { if } n \text { is even } \\
\operatorname{ord}_{2}\left(\left[\frac{n}{2}\right]\right)+1 & \text { otherwise. }
\end{array}\right.
$$

The assertion is an immediate consequence of this fact.

The next lemma, combined with Lemma 2.4, plainly explains the difference between the cases where $p>2$ and $p=2$. 
Lemma 2.6 Let $m$ be a positive integer. Then

$$
h_{n}\left(C_{2^{m}} ; C_{2^{m+1}}\right) \equiv 2^{m n} h_{n}\left(C_{2}\right) \quad \bmod 2^{m n+[n / 2]-[n / 4]+1} .
$$

Moreover, if $0 \leq n \leq 3$, then

$$
h_{n}\left(C_{2^{m}} ; C_{2^{m+1}}\right)=2^{m n} h_{n}\left(C_{2}\right) .
$$

Proof. We keep the notation of Lemma 2.3, and prove the first assertion. For each integer $k$ with $3 \leq k \leq m$, we have

$$
r_{2}(k)=2^{k}-1-k>2^{k-2} .
$$

Moreover, if $j_{0}+2 j_{1}+\cdots+2^{m} j_{m}=n$, then

$$
\left[\frac{n}{2}\right]-\left[\frac{n}{4}\right]=\left[\frac{j_{0}+2 j_{1}}{2}\right]-\left[\frac{j_{0}+2 j_{1}}{4}\right]+\sum_{k=2}^{m} 2^{k-2} j_{k} .
$$

Hence Lemma 2.3 yields

$$
\begin{aligned}
& h_{n}\left(C_{2^{m}} ; C_{2^{m+1}}\right) \\
& =\sum_{i=0}^{n} \sum_{\substack{j_{0}+2 j_{1}=i \\
2^{2} j_{2}+\cdots+2^{m} j_{m}=n-i}} \frac{i !}{j_{0} ! 2^{j_{1}} j_{1} !} \cdot \frac{n !}{i !(n-i) !} \cdot \frac{(n-i) !}{\prod_{k=2}^{m} 2^{k j_{k}} j_{k} !} 2^{m n+\sum_{k=0}^{m} r_{2}(k) j_{k}} \\
& =\sum_{i=0}^{n} \frac{n !}{i !(n-i) !} h_{i}\left(C_{2}\right) \sum_{2^{2} j_{2}+\cdots+2^{m} j_{m}=n-i} \frac{(n-i) !}{\prod_{k=2}^{m} 2^{k j_{k}} j_{k} !} 2^{m n+\sum_{k=2}^{m} r_{2}(k) j_{k}} \\
& \equiv 2^{m n} h_{n}\left(C_{2}\right) \quad \bmod 2^{m n+[n / 2]-[n / 4]+1} \text {. }
\end{aligned}
$$

Here

$$
h_{i}\left(C_{2}\right)=\sum_{j_{0}+2 j_{1}=i} \frac{i !}{j_{0} ! 2^{j_{1}} j_{1} !}
$$

by (A) with $d=2$ and $H=\left\{e_{H}\right\}$,

$$
\operatorname{ord}_{2}\left(h_{i}\left(C_{2}\right)\right) \geq\left[\frac{i}{2}\right]-\left[\frac{i}{4}\right]
$$

by [12] (see also Theorem 2.1), and

$$
\operatorname{ord}_{2}\left(\frac{(n-i) !}{2^{2 j_{2}} j_{2} !}\right)=\operatorname{ord}_{2}\left(\frac{\left(2^{2} j_{2}\right) !}{2^{2 j_{2}} j_{2} !}\right)=j_{2} \geq 1
$$

if $2^{2} j_{2}=n-i \geq 4$. This proves the lemma.

The next theorem is stated in [9, Theorem 4.2]. 
Theorem 2.2 Suppose that $p=2$ and $u=v \geq 1$. Let $y_{n}=\left[n / 2^{u+1}\right]$ for each nonnegative integer $n$. Then

$$
\operatorname{ord}_{2}\left(h_{n}(P)\right) \geq \tau_{\lambda}^{2}(n)
$$

and

$$
h_{n}(P) \equiv \frac{n !}{2^{y_{n}} y_{n} !\left(n-2^{u+1} y_{n}\right) !} h_{y_{n}}\left(C_{2}\right) h_{n-2^{u+1} y_{n}}(P) \quad \bmod 2^{\tau_{\lambda}^{2}(n)+1} .
$$

Proof. Recall that $\tau_{\lambda}^{2}(n)=f_{2}^{u}(n)+\left[y_{n} / 2\right]-\left[y_{n} / 4\right]$. By Theorem 2.1, we may assume that $y_{n} \geq 1$. We use induction on $y_{n}$. If $y_{n}=1$, then the first assertion is a consequence of Theorem 2.1. Assume that $y_{n}=k+2 l+4 m$, where $k, l \in\{0,1\}$. We have $y_{n}-2\left[y_{n} / 2\right]+\left[y_{n} / 4\right]=k+m$. Hence, if $y_{n} \neq 2$, then by Theorem 2.1 and Lemma 2.1,

$$
\begin{aligned}
\operatorname{ord}_{2}\left(h_{n}\left(C_{2^{u+1}} \times C_{2^{u}}\right)\right) & \geq f_{2}^{u+1}(n)-\left[\frac{n}{2^{u+2}}\right] \\
& =f_{2}^{u}(n)+y_{n}-\left[\frac{y_{n}}{2}\right] \\
& \geq \tau_{\lambda}^{2}(n)+1
\end{aligned}
$$

and

$$
\sum_{i=0}^{y_{n}} \frac{n ! h_{i}\left(C_{2^{u}} ; C_{2^{u+1}}\right)}{2^{(u+1) i} i !} \cdot \frac{h_{n-2^{u+1} i}(P)}{\left(n-2^{u+1} i\right) !} \equiv 0 \quad \bmod 2^{\tau_{\lambda}^{2}(n)+1} .
$$

In particular, if $y_{n}=1$, then it follows from Lemma 2.6 that

$$
h_{n}(P) \equiv-\frac{n !}{2\left(n-2^{u+1}\right) !} h_{n-2^{u+1}}(P) \quad \bmod 2^{\tau_{\lambda}^{2}(n)+1} .
$$

Suppose that $y_{n}=2$. Then by the above fact and Lemmas 2.1 and 2.6,

$$
\begin{aligned}
& h_{n}\left(C_{2^{u+1}} \times C_{2^{u}}\right) \\
& \quad=h_{n}(P)+\frac{n !}{2\left(n-2^{u+1}\right) !} h_{n-2^{u+1}}(P)+\frac{n !}{2^{2}\left(n-2^{u+2}\right) !} h_{n-2^{u+2}}(P) \\
& \quad \equiv h_{n}(P) \quad \bmod 2^{\tau_{\lambda}^{2}(n)+1} .
\end{aligned}
$$

This, combined with Theorem 2.1 and the fact that

$$
h_{n-2^{u+2}}(P)=h_{n-2^{u+2}}\left(C_{2^{u+1}} \times C_{2^{u}}\right),
$$


shows that $\operatorname{ord}_{2}\left(h_{n}(P)\right) \geq \tau_{\lambda}^{2}(n)$ and

$$
h_{n}(P) \equiv \frac{n !}{2^{2}\left(n-2^{u+2}\right) !} h_{n-2^{u+2}}(P) \quad \bmod 2^{\tau_{\lambda}^{2}(n)+1} .
$$

Suppose now that $y_{n} \geq 3$. By Lemma 2.2 and the inductive assumption,

$$
\begin{aligned}
\operatorname{ord}_{2}\left(\frac{n !}{2^{(u+1) i} i !\left(n-2^{u+1} i\right) !} h_{n-2^{u+1} i}(P)\right) \\
\geq f_{2}^{u}\left(2^{u+1} i\right)-u i+\left[\frac{y_{n}}{2}\right]-\left[\frac{i}{2}\right]-\left[\frac{y_{n}-i}{2}\right] \\
+f_{2}^{u}\left(n-2^{u+1} i\right)+\left[\frac{y_{n}-i}{2}\right]-\left[\frac{y_{n}-i}{4}\right] \\
=f_{2}^{u}(n)-u i+\left[\frac{y_{n}}{2}\right]-\left[\frac{i}{2}\right]-\left[\frac{y_{n}-i}{4}\right]
\end{aligned}
$$

for any integer $i$ with $1 \leq i \leq y_{n}$. Hence the first assertion follows from Theorem 2.1, Lemma 2.6, and (F). Moreover, it follows from the inductive assumption that

$$
\begin{aligned}
& h_{n}(P) \equiv-\sum_{i=1}^{y_{n}} \frac{n ! h_{i}\left(C_{2^{u}} ; C_{2^{u+1}}\right)}{2^{(u+1) i} i !} \cdot \frac{h_{n-2^{u+1} i}(P)}{\left(n-2^{u+1} i\right) !} \\
& \equiv-\sum_{i=1}^{y_{n}} \frac{n ! h_{i}\left(C_{2}\right)}{2^{i} i !} \cdot \frac{h_{y_{n}-i}\left(C_{2}\right)}{2^{y_{n}-i}\left(y_{n}-i\right) !} \cdot \frac{h_{n-2^{u+1} y_{n}}(P)}{\left(n-2^{u+1} y_{n}\right) !} \\
& \equiv\left\{-\sum_{i=1}^{y_{n}} y_{n} ! \frac{h_{i}\left(C_{2}\right)}{i !} \cdot \frac{h_{y_{n}-i}\left(C_{2}\right)}{\left(y_{n}-i\right) !}\right\} \frac{n !}{2^{y_{n}} y_{n} !\left(n-2^{u+1} y_{n}\right) !} h_{n-2^{u+1} y_{n}}(P) \\
& \bmod 2^{\tau_{\lambda}^{2}(n)+1} .
\end{aligned}
$$

Thus the second assertion follows from (E) and Lemma 2.5. This completes the proof.

\section{The number of subgroups of a finite abelian $p$-group}

Recall that $\lambda=\left(\lambda_{1}, \lambda_{2}, \ldots\right)$. We must study the properties of $\alpha_{\lambda}(i ; p)$. Let $a_{i, j}, i, j \in \mathbb{Z}$, denote nonnegative integers such that $\alpha_{\lambda}(i ; p)=\sum_{j} a_{i, j} p^{j}$, which depend only on $\lambda$ and $i$. When $i$ or $j$ is a negative integer, we consider $a_{i, j}=0$. By the duality of finite abelian $p$-groups,

$$
\alpha_{\lambda}(i ; p)=\alpha_{\lambda}(s-i ; p)
$$


whence $a_{i, j}=a_{s-i, j}$. It is clear that $\alpha_{\lambda}(0 ; p)=\alpha_{\lambda}(s ; p)=1$. Furthermore, if $\lambda_{r} \geq 1$ and if $\lambda_{r+1}=0$, then $\alpha_{\lambda}(1 ; p)=\alpha_{\lambda}(s-1 ; p)=1+p+\cdots+p^{r-1}$.

Definition 3.1 We define the partition $\widehat{\lambda}$ of $s-\lambda_{1}$ by $\widehat{\lambda}=\left(\lambda_{2}, \lambda_{3}, \ldots\right)$, and define $\widehat{a}_{i, j}, i, j \in \mathbb{Z}$, to be nonnegative integers such that $\alpha_{\widehat{\lambda}}(i ; p)=\sum_{j} \widehat{a}_{i, j} p^{j}$, which depend only on $\widehat{\lambda}$ and $i$.

The following lemma is useful for an investigation into the coefficients $a_{i, j}$.

Lemma 3.1 We have

$$
a_{i, j}-a_{i-1, j}=\widehat{a}_{i, j-i}-\widehat{a}_{s-i+1, j-s+i-1} .
$$

Proof. We may assume that $s \geq 1$. Suppose that $k$ is the largest number such that $\lambda_{k}=\lambda_{1}$, and define the partition $\widetilde{\lambda}$ of $s-1$ by

$$
\widetilde{\lambda}=\left(\lambda_{1}, \ldots, \lambda_{k-1}, \lambda_{k}-1, \lambda_{k+1}, \ldots\right) .
$$

Then it follows from [15, Theorem 1, Corollary] that

$$
\begin{aligned}
\alpha_{\lambda}(i ; p) & =\alpha_{\widetilde{\lambda}}(i ; p)+p^{s-i} \alpha_{\widehat{\lambda}}\left(i-\lambda_{1} ; p\right) \\
& =\alpha_{\widetilde{\lambda}}(i ; p)+p^{s-i} \alpha_{\widehat{\lambda}}(s-i ; p)
\end{aligned}
$$

and

$$
\alpha_{\lambda}(i ; p)=\alpha_{\widetilde{\lambda}}(i-1 ; p)+p^{i} \alpha_{\widehat{\lambda}}(i ; p) .
$$

Here the former yields

$$
\alpha_{\tilde{\lambda}}(i-1 ; p)=\alpha_{\lambda}(i-1 ; p)-p^{s-i+1} \alpha_{\widehat{\lambda}}(s-i+1 ; p),
$$

and the latter yields

$$
\alpha_{\widetilde{\lambda}}(i-1 ; p)=\alpha_{\lambda}(i ; p)-p^{i} \alpha_{\widehat{\lambda}}(i ; p) .
$$

By these equations, we have

$$
\alpha_{\lambda}(i ; p)-\alpha_{\lambda}(i-1 ; p)=p^{i} \alpha_{\widehat{\lambda}}(i ; p)-p^{s-i+1} \alpha_{\widehat{\lambda}}(s-i+1 ; p),
$$

which is shown in [16]. Now the assertion is an immediate consequence of this equation. This proves the lemma.

Using Lemma 3.1 we get several properties of the coefficients $a_{i, j}, i, j \in \mathbb{Z}$. 
Proposition 3.1 Put $t=s-\lambda_{1}$. The following statements hold.

(1) If $i+j \leq s$, then $a_{i, j} \geq a_{i-1, j}$.

(2) If $i+j \leq s$ and if $i>j$, then $a_{i, j}=a_{i-1, j}$.

(3) If $i+j \leq s$ and if $j \leq t$, then $a_{i, j}-a_{i-1, j-1} \geq a_{i-1, j}-a_{i-2, j-1}$.

(4) If $0 \leq j \leq \min \{t,[s / 2]\}$, then $a_{j, j}=a_{j-1, j}+1$.

(5) If $0<j<\min \{t,[(s+1) / 2]\}$ and if $\lambda_{3} \geq 1$, then $a_{j, j+1}=a_{j-1, j+1}+1$.

(6) Assume that $t<[(s+1) / 2]$. If $t<i<s-t+1$, then $a_{i, j}=a_{i-1, j}$ for any integer $j$.

(7) Assume that $t<[(s+1) / 2]$. If $j>t$, then $a_{t, j}=a_{t-1, j}$.

Proof. The assertion (1) follows from Lemma 3.1. For, if $i+j \leq s$, then $\widehat{a}_{s-i+1, j-s+i-1}=0$ because $j-s+i \leq 0$, and hence $a_{i, j}-a_{i-1, j}=\widehat{a}_{i, j-i}$ by Lemma 3.1. In the proof of (2)-(7), we use this fact without notice.

(2) If $i+j \leq s$ and if $i>j$, then $a_{i, j}-a_{i-1, j}=\widehat{a}_{i, j-i}=0$.

(3) Assume that $i+j \leq s$ and that $j \leq t$. Then

$$
\begin{aligned}
\left(a_{i, j}-a_{i-1, j-1}\right)-\left(a_{i-1, j}-a_{i-2, j-1}\right) & =\left(a_{i, j}-a_{i-1, j}\right)-\left(a_{i-1, j-1}-a_{i-2, j-1}\right) \\
& =\widehat{a}_{i, j-i}-\widehat{a}_{i-1, j-i} .
\end{aligned}
$$

Since $i+(j-i) \leq t$, it follows from (1) that $\widehat{a}_{i, j-i} \geq \widehat{a}_{i-1, j-i}$. Hence we have $a_{i, j}-a_{i-1, j-1} \geq a_{i-1, j}-a_{i-2, j-1}$.

(4) Applying (2) to $\alpha_{\widehat{\lambda}}(i ; p), i \in \mathbb{Z}$, we get $\widehat{a}_{j, 0}=1$ for any $j$ with $0 \leq j \leq t$. Hence, if $0 \leq j \leq \min \{t,[s / 2]\}$, then $a_{j, j}=a_{j-1, j}+\widehat{a}_{j, 0}=a_{j-1, j}+1$.

(5) Assume that $\lambda_{3} \geq 1$. If $0<j<t$, then $\widehat{a}_{j, 1}=1$ by (2) and (4) [11]. Hence, if $0<j<\min \{t,[(s+1) / 2]\}$, then $a_{j, j+1}=a_{j-1, j+1}+\widehat{a}_{j, 1}=a_{j-1, j+1}+1$.

(6) We may assume that $i \leq[(s+1) / 2]$. Let $j$ be any integer. If $t<i$, then $\widehat{a}_{i, j-i}=\widehat{a}_{s-i+1, j-s+i-1}=0$ because $t<i \leq s-i+1$, and hence $a_{i, j}=a_{i-1, j}$ by Lemma 3.1.

(7) Let $j$ be any integer. Since $t<s-t+1$, it follows that $\widehat{a}_{s-t+1, j-s+t-1}=0$. Hence $a_{t, j}=a_{t-1, j}+\widehat{a}_{t, j-t}$ by Lemma 3.1. Moreover, if $j>t$, then $\widehat{a}_{t, j-t}=0$, and thereby, $a_{t, j}=a_{t-1, j}$. 
Remark 3.1 The assertion (1) of Proposition 3.1 is a part of [1, Theorem] (see Section 1). According to [16], Lemma 3.1 enables us to get [1, Theorem]. Also, (2), (4), and (6) of Proposition 3.1 yield [1, Note]:

$$
\begin{array}{ll}
\alpha_{\lambda}(i ; p)=\alpha_{\lambda}(i-1 ; p) & \text { if } \quad t<i \leq[(s+1) / 2], \\
\alpha_{\lambda}(i ; p) \equiv \alpha_{\lambda}(i-1 ; p)+p^{i} \quad \bmod p^{i+1} & \text { if } \quad 0 \leq i \leq \min \{t,[s / 2]\} .
\end{array}
$$

We apply Proposition 3.1 to the following explicit result (see also [19, Proposition 5.3]), which is used in [9].

Proposition 3.2 Suppose that $\lambda=(u, v, 0, \ldots)$. Then

$$
\alpha_{\lambda}(i ; p)= \begin{cases}1+p+\cdots+p^{i} & \text { if } \quad 0 \leq i<v \\ 1+p+\cdots+p^{v} & \text { if } \quad v \leq i \leq u \\ 1+p+\cdots+p^{s-i} & \text { if } \quad u<i \leq s\end{cases}
$$

Proof. We determine $a_{i, j}, i, j \in \mathbb{Z}$. Since $\widehat{\lambda}$ is the type of $C_{p^{v}}$, it follows that $\widehat{a}_{s-i+1, j-s+i-1}=0$ for any $j$ greater than $v$. If $i \leq j \leq v$, then $i+j \leq s$, and thereby, $\widehat{a}_{s-i+1, j-s+i-1}=0$. Hence, if $i<j$, then Lemma 3.1 yields $a_{i, j}-a_{i-1, j}=\widehat{a}_{i, j-i}=0$. Thus, if $i<j$, then $a_{i, j}=a_{i-1, j}=\cdots=a_{0, j}=0$. Now we may assume that $j \leq i$. If $v<j \leq i \leq u$, then $a_{v, j}=0$, and hence $a_{i, j}=\cdots=a_{v, j}=0$ by (6) of Proposition 3.1. If $0 \leq j \leq v$ and if $j \leq i \leq u$, then $a_{i, j}=\cdots=a_{j, j}=a_{j-1, j}+1=1$ by (2) and (4) of Proposition 3.1. We have thus determined $a_{i, j}$ in the case where either $i<j$ or $0 \leq j \leq i \leq u$. Now the proposition follows from the duality of finite abelian $p$-groups. This completes the proof.

\section{A decomposition of the exponential formula}

In this section we give a decomposition of $E_{P}(X)$ for any finite abelian $p$-group $P$. Recall that $v=\min \left\{s-\lambda_{1},[s / 2]\right\}$. We start with two definitions:

Definition 4.1 For each integer $m$ with $0 \leq m \leq s+1$, put

$$
\varphi_{\lambda}^{m}(X)= \begin{cases}\sum_{j=m}^{v} \sum_{k=j}^{s}\left(a_{s-k, j}-a_{s-k-j+m-1, m-1}\right) \frac{X^{p^{k}}}{p^{k-j}}+\varphi_{\lambda}^{v+1}(X) & \text { if } 0 \leq m \leq v, \\ \sum_{j=m}^{s} \sum_{k=j}^{s} a_{s-k, j} \frac{X^{p^{k}}}{p^{k-j}} & \text { if } v<m \leq s, \\ 0 & \text { if } m=s+1 .\end{cases}
$$

We define $\Phi_{\lambda}(X):=\exp \left(\varphi_{\lambda}^{0}(X)\right)$. 
Definition 4.2 For each pair $(l, m) \in \mathbb{Z} \times \mathbb{Z}$ of nonnegative integers $l$ and $m$ with $m \leq s$, put $b_{l, m}=a_{l, m}-a_{l-1, m-1}$, and define

$$
c_{l, m}:=\left\{\begin{array}{ll}
b_{l, m}-b_{l-1, m} & \text { if } 0 \leq l \leq s-m \quad \text { and if } \quad 0 \leq m \leq v, \\
a_{l, m}-a_{l-1, m} & \text { if } 0 \leq l \leq s-m \\
a_{m, l} & \text { if } l>s-m .
\end{array} \text { and if } \quad v<m \leq s,\right.
$$

There are only finitely many pairs $(l, m)$ such that $c_{l, m} \neq 0$. The properties of $c_{l, m}$ play an important role in the proof of Theorem 1.1.

Proposition 4.1 The integer $c_{l, m}$ is nonnegative for any nonnegative integers $l$ and $m$ with $m \leq s$. In particular, $c_{0,0}=1$, and $c_{0, m}=0$ if $m \geq 1$.

Proof. Let $l$ and $m$ be nonnegative integers with $m \leq s$. If $l>s-m$, then $c_{l, m}=a_{m, l} \geq 0$. If $l \leq s-m$, then the first assertion follows from (1) and (3) of Proposition 3.1. The last assertion is clear. This completes the proof.

Now we are ready to show the following.

Theorem 4.1 Let $P$ be a finite abelian p-group of type $\lambda$. Then $E_{P}(X)$ and $\Phi_{\lambda}(X)$ are decomposed as

$$
\begin{aligned}
& E_{P}(X)=\Phi_{\lambda}(X) \prod_{m=0}^{s} \prod_{l=s-m+1}^{\infty} \exp \left(p^{l+m-s} X^{p^{s-m}}\right)^{c_{l, m}} \\
& \Phi_{\lambda}(X)=\prod_{m=0}^{v} \prod_{l=0}^{m} E_{C_{p^{u-l}} \times C_{p^{v-m}}}\left(X^{p^{m}}\right)^{c_{l, m}} \prod_{m=v+1}^{s} \prod_{l=0}^{s-m} E_{C_{p^{s-l-m}}}\left(X^{p^{m}}\right)^{c_{l, m}} .
\end{aligned}
$$

Proof. The number of subgroups of index $p^{k}$ in $P$ is $\alpha_{\lambda}(s-k ; p)$, whence $E_{P}(X)=\exp \left(\sum_{k=0}^{s} \alpha_{\lambda}(s-k ; p) X^{p^{k}} / p^{k}\right)$. Furthermore, we have

$$
\begin{aligned}
\sum_{k=0}^{s} \alpha_{\lambda}(s-k ; p) \frac{X^{p^{k}}}{p^{k}} & =\sum_{k=0}^{s} \sum_{j=0}^{k} a_{s-k, j} \frac{X^{p^{k}}}{p^{k-j}}+\sum_{k=0}^{s} \sum_{j=k+1}^{\infty} a_{s-k, j} p^{j-k} X^{p^{k}} \\
& =\varphi_{\lambda}^{0}(X)+\sum_{m=0}^{s} \sum_{l=s-m+1}^{\infty} a_{m, l} p^{l+m-s} X^{p^{s-m}} .
\end{aligned}
$$


Then the first decomposition of $E_{P}(X)$ clearly holds, and hence it remains to show the decomposition of $\Phi_{\lambda}(X)$. By Definitions 4.1 and 4.2, we obtain

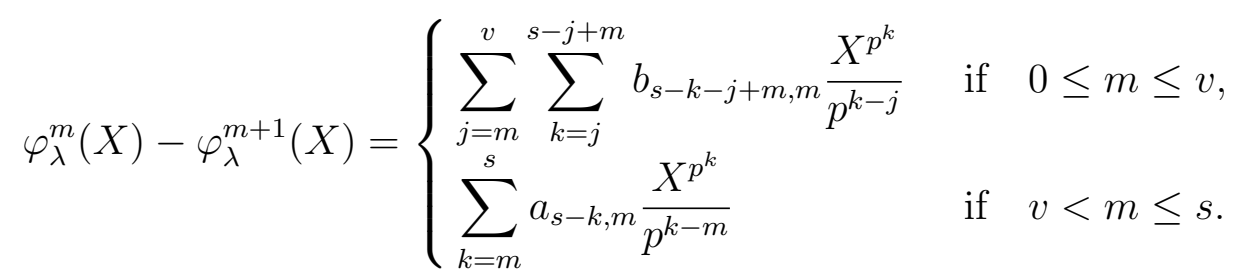

If $m<l \leq s-m$, then $a_{l, m}=a_{l-1, m}$ and $a_{l-1, m-1}=a_{l-2, m-1}$ by (2) of Proposition 3.1, which forces $b_{l, m}=b_{l-1, m}$. Hence, for any integer $m$ with $0 \leq m \leq v$,

$$
\begin{aligned}
\varphi_{\lambda}^{m}(X)-\varphi_{\lambda}^{m+1}(X) & =\sum_{j=m}^{v} \sum_{k=j}^{s-j+m} b_{s-k-j+m, m} \frac{X^{p^{k}}}{p^{k-j}} \\
& =\sum_{j=m}^{v} \sum_{k=j}^{s-j+m}\left(b_{s-k-j+m, m}-b_{s-k-j+m-1, m}\right) \sum_{i=j}^{k} \frac{X^{p^{i}}}{p^{i-j}} \\
& =\sum_{j=m}^{v} \sum_{l=0}^{m}\left(b_{l, m}-b_{l-1, m}\right) \sum_{i=j}^{s-l-j+m} \frac{X^{p^{i}}}{p^{i-j}} \\
& =\sum_{l=0}^{m} c_{l, m} \sum_{j=0}^{v-m} \sum_{i=j}^{s-l-m-j} p^{j} \frac{X^{p^{m+i}}}{p^{i}} .
\end{aligned}
$$

Moreover, for any integer $m$ with $v<m \leq s$,

$$
\begin{aligned}
\varphi_{\lambda}^{m}(X)-\varphi_{\lambda}^{m+1}(X) & =\sum_{k=m}^{s} a_{s-k, m} \frac{X^{p^{k}}}{p^{k-m}} \\
& =\sum_{k=m}^{s}\left(a_{s-k, m}-a_{s-k-1, m}\right) \sum_{i=m}^{k} \frac{X^{p^{i}}}{p^{i-m}} \\
& =\sum_{l=0}^{s-m} c_{l, m} \sum_{i=0}^{s-l-m} \frac{X^{p^{m+i}}}{p^{i}} .
\end{aligned}
$$

Now, since $\varphi_{\lambda}^{0}(X)=\sum_{m=0}^{s}\left(\varphi_{\lambda}^{m}(X)-\varphi_{\lambda}^{m+1}(X)\right)$, we obtain

$$
\varphi_{\lambda}^{0}(X)=\sum_{m=0}^{v} \sum_{l=0}^{m} c_{l, m} \sum_{j=0}^{v-m} \sum_{i=j}^{s-l-m-j} p^{j} \frac{X^{p^{m+i}}}{p^{i}}+\sum_{m=v+1}^{s} \sum_{l=0}^{s-m} c_{l, m} \sum_{i=0}^{s-l-m} \frac{X^{p^{m+i}}}{p^{i}} .
$$


Here Proposition 3.2 implies that, if $0 \leq m \leq v$ and if $0 \leq l \leq m$, then

$$
E_{C_{p^{u-l}} \times C_{p^{v-m}}}\left(X^{p^{m}}\right)=\exp \left(\sum_{j=0}^{v-m} \sum_{i=j}^{s-l-m-j} p^{j} \frac{X^{p^{m+i}}}{p^{i}}\right) .
$$

Furthermore, if $0 \leq l \leq s-m$, then

$$
E_{C_{p^{s-l-m}}}\left(X^{p^{m}}\right)=\exp \left(\sum_{i=0}^{s-l-m} \frac{X^{p^{m+i}}}{p^{i}}\right) .
$$

Hence the decomposition of $\Phi_{\lambda}(X)$ holds. We have thus proved the theorem.

Example 4.1 Suppose that $\lambda=(1,1,1,1,1,1,0, \ldots)$. Then $u=v=3$, and the table of nonzero $a_{i, j}, i, j \in \mathbb{Z}$, is the following.

\begin{tabular}{c|cccccccccc}
$i$ & $a_{i, 0}$ & $a_{i, 1}$ & $a_{i, 2}$ & $a_{i, 3}$ & $a_{i, 4}$ & $a_{i, 5}$ & $a_{i, 6}$ & $a_{i, 7}$ & $a_{i, 8}$ & $a_{i, 9}$ \\
\hline 0 & 1 & & & & & & & & & \\
1 & 1 & 1 & 1 & 1 & 1 & 1 & & & & \\
2 & 1 & 1 & 2 & 2 & 3 & 2 & 2 & 1 & 1 & \\
3 & 1 & 1 & 2 & 3 & 3 & 3 & 3 & 2 & 1 & 1 \\
4 & 1 & 1 & 2 & 2 & 3 & 2 & 2 & 1 & 1 & \\
5 & 1 & 1 & 1 & 1 & 1 & 1 & & & & \\
6 & 1 & & & & & & & & &
\end{tabular}

For each $(l, m) \in \mathbb{Z} \times \mathbb{Z}$ with $0 \leq m \leq 6$ and $0 \leq l \leq 6-m$,

$$
c_{l, m}= \begin{cases}1 & \text { if } \quad(l, m)=(0,0), \quad(1, m) \quad \text { where } \quad 2 \leq m \leq 5 \\ 2 & \text { if } \quad(l, m)=(2,4), \\ 0 & \text { otherwise. }\end{cases}
$$

Consequently, we have

$$
\Phi_{\lambda}(X)=E_{C_{p^{3}} \times C_{p^{3}}}(X) E_{C_{p^{2}} \times C_{p}}\left(X^{p^{2}}\right) E_{C_{p^{2}}}\left(X^{p^{3}}\right) E_{C_{p}}\left(X^{p^{4}}\right) \exp \left(X^{p^{4}}\right)^{2} \exp \left(X^{p^{5}}\right) .
$$

In order to prove Theorem 1.1, we need further information about $c_{l, m}$. Recall that $u=\max \left\{\lambda_{1},[(s+1) / 2]\right\}=s-v$.

Lemma 4.1 Suppose that $0 \leq m \leq v$. Unless $\lambda_{3} \geq 1$ and $(l, m)=(1,2)$, $c_{l, m}=0$ for any positive integer $l$ with $m-1 \leq l \leq s-m$. If $\lambda_{3} \geq 1$ and if $m=2$, then $c_{1,2}=1$. 
Proof. If $\lambda_{3} \geq 1$ and if $m=2$, then $c_{1,2}=b_{1,2}-b_{0,2}=a_{1,2}=1$. Thus the second assertion holds. We prove the first one.

If $m<l \leq s-m$, then $a_{l, m}=a_{l-1, m}$ and $a_{l-1, m-1}=a_{l-2, m-1}$ by (2) of Proposition 3.1, which yields $c_{l, m}=0$. Also, if $m \geq 1$, then by (4) of Proposition 3.1, $a_{m, m}-a_{m-1, m}=a_{m-1, m-1}-a_{m-2, m-1}=1$, and hence $c_{m, m}=0$. Moreover, if $\lambda_{3}=0$ and if $m \geq 2$, then $c_{m-1, m}=0$ by Proposition 3.2. Now we assume that $\lambda_{3} \geq 1$ and $m \geq 3$. Then, since $m-1<v=\min \left\{s-\lambda_{1},[s / 2]\right\}$, (5) of Proposition 3.1 implies that $a_{m-1, m}-a_{m-2, m}=a_{m-2, m-1}-a_{m-3, m-1}=1$. Hence we have $c_{m-1, m}=0$, as desired. Thus the first assertion holds.

Lemma 4.2 Suppose that $v<m \leq u$. If $u=\lambda_{1}$, then $c_{l, m}=0$ for any integer $l$ with $v \leq l \leq s-m$. If $u>\lambda_{1}$, then $u=v+1$ and $c_{v, v+1}=1$.

Proof. Since $v<u$, it follows that $v<[(s+1) / 2]$. Hence, if $u=\lambda_{1}$, then $s-\lambda_{1}=v<[(s+1) / 2]$ and $s-\lambda_{1} \leq s-m<\lambda_{1}$, and thereby, it follows from (6) and (7) of Proposition 3.1 that $a_{v-1, m}=a_{v, m}=\cdots=a_{s-m, m}$. Thus, if $u=\lambda_{1}$, then $c_{l, m}=0$ for any integer $l$ with $v \leq l \leq s-m$. Assume that $u>\lambda_{1}$. Then $\lambda_{3} \geq 1$ and $u=[(s+1) / 2]$. Furthermore, $0<v<\min \left\{s-\lambda_{1},[(s+1) / 2]\right\}$ and $u=v+1$, because $v=s-u<s-\lambda_{1}$ and $[s / 2]=v<u=[(s+1) / 2]$. Hence, by (5) of Proposition 3.1, $c_{v, v+1}=a_{v, v+1}-a_{v-1, v+1}=1$. This completes the proof.

Example 4.2 Suppose that $\lambda=(5,1,1,1,0, \ldots)$. Then $u=5=\lambda_{1}$ and $v=3$, and the table of nonzero $a_{i, j}, i, j \in \mathbb{Z}$, is the following.

\begin{tabular}{c|ccccc}
$i$ & $a_{i, 0}$ & $a_{i, 1}$ & $a_{i, 2}$ & $a_{i, 3}$ & $a_{i, 4}$ \\
\hline 0 & 1 & & & & \\
1 & 1 & 1 & 1 & 1 & \\
2 & 1 & 1 & 2 & 2 & 1 \\
3 & 1 & 1 & 2 & 3 & 1 \\
4 & 1 & 1 & 2 & 3 & 1 \\
5 & 1 & 1 & 2 & 3 & 1 \\
6 & 1 & 1 & 2 & 2 & 1 \\
7 & 1 & 1 & 1 & 1 & \\
8 & 1 & & & &
\end{tabular}

For each $(l, m) \in \mathbb{Z} \times \mathbb{Z}$ with $0 \leq m \leq 8$ and $0 \leq l \leq 8-m$,

$$
c_{l, m}= \begin{cases}1 & \text { if } \quad(l, m)=(0,0), \quad(1,2), \quad(1,3), \quad(2,4), \\ 0 & \text { otherwise }\end{cases}
$$


Hence we have

$$
\Phi_{\lambda}(X)=E_{C_{p^{5}} \times C_{p^{3}}}(X) E_{C_{p^{4}} \times C_{p}}\left(X^{p^{2}}\right) E_{C_{p^{4}}}\left(X^{p^{3}}\right) E_{C_{p^{2}}}\left(X^{p^{4}}\right) .
$$

Example 4.3 Suppose that $\lambda=(2,2,1,0, \ldots)$. Then $u=3>\lambda_{1}=2$ and $v=2$, and the table of nonzero $a_{i, j}, i, j \in \mathbb{Z}$, is the following.

\begin{tabular}{c|cccc}
$i$ & $a_{i, 0}$ & $a_{i, 1}$ & $a_{i, 2}$ & $a_{i, 3}$ \\
\hline 0 & 1 & & & \\
1 & 1 & 1 & 1 & \\
2 & 1 & 1 & 2 & 1 \\
3 & 1 & 1 & 2 & 1 \\
4 & 1 & 1 & 1 & \\
5 & 1 & & &
\end{tabular}

For each $(l, m) \in \mathbb{Z} \times \mathbb{Z}$ with $0 \leq m \leq 5$ and $0 \leq l \leq 5-m$,

$$
c_{l, m}= \begin{cases}1 & \text { if } \quad(l, m)=(0,0), \quad(1,2), \quad(2,3), \\ 0 & \text { otherwise }\end{cases}
$$

Now we get

$$
\Phi_{\lambda}(X)=E_{C_{p^{3}} \times C_{p^{2}}}(X) E_{C_{p^{2}}}\left(X^{p^{2}}\right) \exp \left(X^{p^{3}}\right)
$$

\section{The lower bound}

In this section, we denote by $P$ a finite abelian $p$-group of type $\lambda$, and show that $\operatorname{ord}_{p}\left(h_{n}(P)\right) \geq \tau_{\lambda}^{p}(n)$ (cf. Theorem 5.2). First of all, we state a consequence of Theorems 2.1 and 2.2:

Theorem 5.1 If $\lambda_{3}=0$, then $\operatorname{ord}_{p}\left(h_{n}(P)\right) \geq \tau_{\lambda}^{p}(n)$.

In order to generalize this theorem, we set

$$
\Omega_{k}=\left\{(l, m) \in \mathbb{Z} \times \mathbb{Z} \mid 0 \leq l \leq s-m, 0 \leq m \leq \min \{k, s\}, c_{l, m} \neq 0\right\}
$$

for each nonnegative integer $k$, and set

$$
\Omega=\left\{(l, m) \in \mathbb{Z} \times \mathbb{Z} \mid l \geq 0,0 \leq m \leq s, c_{l, m} \neq 0\right\} .
$$

Note that, if $\lambda_{3}=0$, then $\Omega=\{(0,0)\}$ by Proposition 3.2. It follows from Lemma 4.1 that $l \leq m$ for each $(l, m) \in \Omega_{v}$. Hence Theorem 4.1 implies that

$$
E_{P}(X)=\Phi_{\lambda}(X) \prod_{(l, m) \in \Omega-\Omega_{s}} \exp \left(p^{l+m-s} X^{p^{s-m}}\right)^{c_{l, m}}
$$


and

$$
\Phi_{\lambda}(X)=\prod_{(l, m) \in \Omega_{v}} E_{C_{p^{u-l}} \times C_{p^{v-m}}}\left(X^{p^{m}}\right)^{c_{l, m}} \prod_{(l, m) \in \Omega_{s}-\Omega_{v}} E_{C_{p^{s-l-m}}}\left(X^{p^{m}}\right)^{c_{l, m}} .
$$

Let $I$ denote the set consisting of all elements $(l, m, i)$ of $\mathbb{Z} \times \mathbb{Z} \times \mathbb{Z}$ such that $(l, m) \in \Omega$ and $1 \leq i \leq c_{l, m}$. Let $\Gamma_{n}$ be the set of mappings $\sigma$ from $I$ to the set of nonnegative integers such that

$$
\sum_{(l, m) \in \Omega_{s}} \sum_{i=1}^{c_{l, m}} p^{m} \sigma(l, m, i)+\sum_{(l, m) \in \Omega-\Omega_{s}} \sum_{i=1}^{c_{l, m}} p^{s-m} \sigma(l, m, i)=n .
$$

Definition 5.1 For each element $\sigma$ of $\Gamma_{n}$, put

$$
h_{n}^{\sigma}(P)=n ! \prod_{(l, m) \in \Omega} \prod_{i=1}^{c_{l, m}} \frac{g_{l, m}(\sigma(l, m, i))}{\sigma(l, m, i) !}
$$

where

$$
g_{l, m}(n)= \begin{cases}h_{n}\left(C_{p^{u-l}} \times C_{p^{v-m}}\right) & \text { if } \quad(l, m) \in \Omega_{v}, \\ h_{n}\left(C_{p^{s-l-m}}\right) & \text { if } \quad(l, m) \in \Omega_{s}-\Omega_{v}, \\ p^{(l+m-s) n} & \text { if } \quad(l, m) \in \Omega-\Omega_{s} .\end{cases}
$$

Now $(\mathrm{G})$ and $(\mathrm{H})$ yield the following.

Proposition 5.1 We have $h_{n}(P)=\sum_{\sigma \in \Gamma_{n}} h_{n}^{\sigma}(P)$.

We wish to show that $\operatorname{ord}_{p}\left(h_{n}^{\sigma}(P)\right) \geq \tau_{\lambda}^{p}(n)$ for all $\sigma \in \Gamma_{n}$.

Definition 5.2 For each nonnegative integer $m$, put

$$
\tau_{\lambda}^{p, m}(n)= \begin{cases}f_{2}^{u-m+2}(n)-\left[\frac{2^{m} n}{2^{u+1}}\right]-\left[\frac{2^{m} n}{2^{u+3}}\right] \quad \text { if } p=2 \quad \text { and if } 2 u=s \geq 2, \\ f_{p}^{u-m}(n)-(2 u-s)\left[\frac{p^{m} n}{p^{u+1}}\right] \quad \text { otherwise. }\end{cases}
$$

Especially, $\tau_{\lambda}^{p}(n)=\tau_{\lambda}^{p, 0}(n)$. Note that $2 u-s=u-v$ and that $2 u=s \geq 2$ if and only if $u=v \geq 1$.

Definition 5.3 For each $\sigma \in \Gamma_{n}$ and for nonnegative integers $j$ and $k$, put

$$
\begin{aligned}
\rho_{k}^{\sigma}(n) & =\sum_{(l, m) \in \Omega_{k}} \sum_{i=1}^{c_{l, m}}\left\{\operatorname{ord}_{p}\left(g_{l, m}(\sigma(l, m, i))\right)-\tau_{\lambda}^{p, m}(\sigma(l, m, i))\right\}, \\
\gamma_{j, k}^{\sigma}(n) & =\left[\frac{n}{p^{j}}\right]-\sum_{(l, m) \in \Omega_{k}} \sum_{i=1}^{c_{l, m}}\left[\frac{p^{m} \sigma(l, m, i)}{p^{j}}\right] .
\end{aligned}
$$


It is evident that $\gamma_{j, k}^{\sigma}(n) \geq 0$ for any nonnegative integers $j$ and $k$.

Lemma 5.1 Let $\sigma \in \Gamma_{n}$. Excepting the case where $p=2$ and $u=v \geq 1$,

$$
\operatorname{ord}_{p}\left(h_{n}^{\sigma}(P)\right) \geq \tau_{\lambda}^{p}(n)+(u-v+1) \gamma_{u+1, u}^{\sigma}(n)+\rho_{u}^{\sigma}(n) .
$$

Furthermore, if $p=2$ and if $u=v \geq 1$, then

$$
\operatorname{ord}_{2}\left(h_{n}^{\sigma}(P)\right) \geq \tau_{\lambda}^{2}(n)+\gamma_{u+1, u+1}^{\sigma}(n)+\rho_{u+1}^{\sigma}(n),
$$

and

$$
\operatorname{ord}_{2}\left(h_{n}^{\sigma}(P)\right) \geq \tau_{\lambda}^{2}(n)+2 \gamma_{u+3, u+1}^{\sigma}(n)+\rho_{u+1}^{\sigma}(n)
$$

Proof. Put $n_{l, m}^{i}=\sigma(l, m, i)$ for each element $(l, m, i)$ of $I$. Since $\operatorname{ord}_{p}(n !)=$ $\sum_{j=1}^{\infty}\left[n / p^{j}\right]$, it follows that $\operatorname{ord}_{p}(n !) \leq n$. Hence

$$
\begin{aligned}
\operatorname{ord}_{p}\left(h_{n}^{\sigma}(P)\right) \geq \sum_{j=1}^{\infty}\left\{\left[\frac{n}{p^{j}}\right]-\right. & \left.\sum_{(l, m) \in \Omega_{s}} \sum_{i=1}^{c_{l, m}}\left[\frac{n_{l, m}^{i}}{p^{j}}\right]\right\} \\
& +\sum_{(l, m) \in \Omega_{s}} \sum_{i=1}^{c_{l, m}} \operatorname{ord}_{p}\left(g_{l, m}\left(n_{l, m}^{i}\right)\right),
\end{aligned}
$$

which yields

$$
\begin{aligned}
& \operatorname{ord}_{p}\left(h_{n}^{\sigma}(P)\right)-f_{p}^{u+1}(n) \\
& \quad \geq \sum_{(l, m) \in \Omega_{u}} \sum_{i=1}^{c_{l, m}}\left\{\operatorname{ord}_{p}\left(g_{l, m}\left(n_{l, m}^{i}\right)\right)-\sum_{j=1}^{u-m+1}\left[\frac{n_{l, m}^{i}}{p^{j}}\right]\right\}+\sum_{j=u+2}^{\infty} \gamma_{j, s}^{\sigma}(n) \\
& \geq \sum_{(l, m) \in \Omega_{u}} \sum_{i=1}^{c_{l, m}}\left\{\tau_{\lambda}^{p, m}\left(n_{l, m}^{i}\right)-f_{p}^{u-m+1}\left(n_{l, m}^{i}\right)\right\}+\rho_{u}^{\sigma}(n) .
\end{aligned}
$$

Now, excepting the case where $p=2$ and $u=v \geq 1$, we obtain

$$
\operatorname{ord}_{p}\left(h_{n}^{\sigma}(P)\right) \geq \tau_{\lambda}^{p}(n)+(u-v+1) \gamma_{u+1, u}^{\sigma}(n)+\rho_{u}^{\sigma}(n) .
$$

Assume that $p=2$ and that $u=v \geq 1$. Then we have $\operatorname{ord}_{2}\left(h_{n}^{\sigma}(P)\right)-f_{2}^{u+2}(n)$

$$
\begin{aligned}
& \geq \sum_{(l, m) \in \Omega_{u+1}} \sum_{i=1}^{c_{l, m}}\left\{\operatorname{ord}_{2}\left(g_{l, m}\left(n_{l, m}^{i}\right)\right)-\sum_{j=1}^{u-m+2}\left[\frac{n_{l, m}^{i}}{2^{j}}\right]\right\}+\sum_{j=u+3}^{\infty} \gamma_{j, s}^{\sigma}(n) \\
& \geq-\sum_{(l, m) \in \Omega_{u+1}} \sum_{i=1}^{c_{l, m}}\left\{\left[\frac{2^{m} n_{l, m}^{i}}{2^{u+1}}\right]+\left[\frac{2^{m} n_{l, m}^{i}}{2^{u+3}}\right]\right\}+\gamma_{u+3, s}^{\sigma}(n)+\rho_{u+1}^{\sigma}(n) .
\end{aligned}
$$


Thus

$$
\operatorname{ord}_{2}\left(h_{n}^{\sigma}(P)\right) \geq \tau_{\lambda}^{2}(n)+\gamma_{u+1, u+1}^{\sigma}(n)+\gamma_{u+3, u+1}^{\sigma}(n)+\gamma_{u+3, s}^{\sigma}(n)+\rho_{u+1}^{\sigma}(n) .
$$

Moreover, since $\gamma_{u+1, u+1}^{\sigma}(n)-\gamma_{u+1, s}^{\sigma}(n) \geq \gamma_{u+3, u+1}^{\sigma}(n)-\gamma_{u+3, s}^{\sigma}(n)$, it follow that

$$
\operatorname{ord}_{2}\left(h_{n}^{\sigma}(P)\right) \geq \tau_{\lambda}^{2}(n)+\gamma_{u+1, s}^{\sigma}(n)+2 \gamma_{u+3, u+1}^{\sigma}(n)+\rho_{u+1}^{\sigma}(n) .
$$

This proves the lemma.

We are trying to show that $\operatorname{ord}_{p}\left(h_{n}^{\sigma}(A)\right) \geq \tau_{\lambda}^{p}(n)$ for all $\sigma \in \Gamma_{n}$. For each nonnegative integer $k$, set

$$
\widehat{\Omega}_{k}=\left\{(l, m) \in \Omega_{k} \mid l \geq 1, m \geq 2\right\} .
$$

Definition 5.4 Put $\kappa_{0,0}(n)=0$, and, for each $(l, m) \in \widehat{\Omega}_{s}$, put

$$
\kappa_{l, m}(n)= \begin{cases}f_{p}^{u-l}(n)-(u-l-v+m)\left[\frac{p^{l} n}{p^{u+1}}\right]-\tau_{\lambda}^{p, m}(n) \quad \text { if } \quad m \leq v, \\ f_{p}^{s-l-m}(n)-(s-l-m)\left[\frac{p^{l+m} n}{p^{s+1}}\right]-\tau_{\lambda}^{p, m}(n) \quad \text { if } \quad v<m \leq s .\end{cases}
$$

Lemma 5.2 We have $\Omega_{s}=\widehat{\Omega}_{s} \cup\{(0,0)\}$ and $\operatorname{ord}_{p}\left(g_{l, m}(n)\right) \geq \kappa_{l, m}(n)+\tau_{\lambda}^{p, m}(n)$ for all $(l, m) \in \Omega_{s}$.

Proof. By Lemmas 4.1 and 4.2, $c_{l, 0}=0$ if $1 \leq l \leq s$, and $c_{l, 1}=0$ if $1 \leq l \leq s-1$. These facts, together with Proposition 4.1, yield $\Omega_{s}=\widehat{\Omega}_{s} \cup\{(0,0)\}$.

It follows from Theorem 5.1 that $\operatorname{ord}_{p}\left(g_{0,0}(n)\right) \geq \tau_{\lambda}^{p, 0}(n)$. If $(l, m) \in \widehat{\Omega}_{v}$, then $u-l>v-m$ because $l<m$ by Lemma 4.1, and hence

$$
\operatorname{ord}_{p}\left(g_{l, m}(n)\right) \geq f_{p}^{u-l}(n)-(u-l-v+m)\left[\frac{p^{l} n}{p^{u+1}}\right]
$$

by Theorem 5.1. Also, if $(l, m) \in \widehat{\Omega}_{s}$ and if $m>v$, then

$$
\operatorname{ord}_{p}\left(g_{l, m}(n)\right) \geq f_{p}^{s-l-m}(n)-(s-l-m)\left[\frac{p^{l+m} n}{p^{s+1}}\right] .
$$

Hence we conclude that $\operatorname{ord}_{p}\left(g_{l, m}(n)\right) \geq \kappa_{l, m}(n)+\tau_{\lambda}^{p, m}(n)$.

Lemma 5.3 Suppose that $\lambda_{3} \geq 1$. Then the following statements hold. 
(1) Assume that either $p>2$ or $u>v$. Let $(l, m) \in \widehat{\Omega}_{u}$. Then $\kappa_{l, m}(n) \geq 0$. If $(l, m) \neq(v, u)$ and if $n \geq p^{u-m+1}$, then $\kappa_{l, m}(n) \geq u-v+1$.

(2) Assume that $p=2$ and that $u=v$. Let $(l, m) \in \widehat{\Omega}_{u+1}$. Then $\kappa_{l, m}(n) \geq 0$. If $(l, m) \neq(1,2)$ and if $n \geq 2^{u-m+1}$, then $\kappa_{l, m}(n) \geq 1$. Furthermore, if $(l, m) \neq(1,2)$ and if $n \geq 2^{u-m+3}$, then $\kappa_{l, m}(n) \geq 3$.

Proof. (1) If $m \leq v$, then $l<m$ by Lemma 4.1, and hence

$$
\begin{aligned}
\kappa_{l, m}(n) & =f_{p}^{u-l}(n)-(u-l-v+m)\left[\frac{p^{l} n}{p^{u+1}}\right]-f_{p}^{u-m}(n)+(u-v)\left[\frac{p^{m} n}{p^{u+1}}\right] \\
& =\sum_{j=u-m+1}^{u-l}\left[\frac{n}{p^{j}}\right]-(m-l)\left[\frac{p^{l} n}{p^{u+1}}\right]+(u-v)\left\{\left[\frac{p^{m} n}{p^{u+1}}\right]-\left[\frac{p^{l} n}{p^{u+1}}\right]\right\} \\
& \geq(u-v+1)\left\{\left[\frac{p^{m} n}{p^{u+1}}\right]-\left[\frac{p^{l} n}{p^{u+1}}\right]\right\} \geq 0 .
\end{aligned}
$$

Moreover, if $m \leq v$ and if $n \geq p^{u-m+1}$, then $\kappa_{l, m}(n) \geq u-v+1$.

Assume that $v<m \leq u$. Since $c_{l, m} \neq 0$ and $l \leq s-m$, it follows from Lemma 4.2 that either $l<v$ or $(l, m)=(v, u)$. By the definition, we have $\kappa_{v, u}(n)=-\tau_{\lambda}^{p, u}(n)=(u-v)[n / p] \geq 0$. On the other hand, if $l<v$, then

$$
\begin{aligned}
\kappa_{l, m}(n) & =f_{p}^{s-l-m}(n)-(s-l-m)\left[\frac{p^{l+m} n}{p^{s+1}}\right]-f_{p}^{u-m}(n)+(u-v)\left[\frac{p^{m} n}{p^{u+1}}\right] \\
& =\sum_{j=u-m+1}^{s-l-m}\left[\frac{n}{p^{j}}\right]-(s-l-m)\left[\frac{p^{m} n}{p^{s-l+1}}\right]+(u-v)\left[\frac{p^{m} n}{p^{u+1}}\right] \\
& \geq(u-v+1)\left[\frac{p^{m} n}{p^{u+1}}\right]-(u-v)\left[\frac{p^{m} n}{p^{u+2}}\right] \geq 0 .
\end{aligned}
$$

Now, if $(l, m) \neq(v, u)$ and if $n \geq p^{u-m+1}$, then $l<v$ and $\kappa_{l, m}(n) \geq u-v+1$.

(2) If $m=u+1$, then by the definition,

$$
\kappa_{l, u+1}(n) \geq-\tau_{\lambda}^{2, u+1}(n)=n-\left[\frac{n}{2}\right]+\left[\frac{n}{2^{2}}\right],
$$

and the assertions follow. Next we assume that $m \leq u$. If $m=2$, then $l=1$ by Lemma 4.1, and

$$
\kappa_{1,2}(n)=\left[\frac{n}{2^{u-1}}\right]-2\left[\frac{n}{2^{u}}\right]+\left[\frac{n}{2^{u+1}}\right] \geq 0 .
$$


Assume that $3 \leq m \leq u$. Then $l \leq m-2$ by Lemma 4.1. If $l \leq m-3$, then

$$
\begin{aligned}
\kappa_{l, m}(n) & =f_{2}^{u-l}(n)-(-l+m)\left[\frac{2^{l} n}{2^{u+1}}\right]-f_{2}^{u-m+2}(n)+\left[\frac{2^{m} n}{2^{u+1}}\right]+\left[\frac{2^{m} n}{2^{u+3}}\right] \\
& =\sum_{j=u-m+3}^{u-l}\left[\frac{n}{2^{j}}\right]-(m-l)\left[\frac{2^{l} n}{2^{u+1}}\right]+\left[\frac{2^{m} n}{2^{u+1}}\right]+\left[\frac{2^{m} n}{2^{u+3}}\right] \\
& \geq\left[\frac{2^{m} n}{2^{u+1}}\right]-\left[\frac{2^{m} n}{2^{u+3}}\right],
\end{aligned}
$$

which yields the assertions. This inequality holds even if $l=m-2$, and hence the lemma follows.

Now the following results are established.

Proposition 5.2 We have $\operatorname{ord}_{p}\left(h_{n}^{\sigma}(P)\right) \geq \tau_{\lambda}^{p}(n)$ for all $\sigma \in \Gamma_{n}$.

Proof. The proposition follows from Lemmas 5.1, 5.2, and 5.3.

Theorem 5.2 We have $\operatorname{ord}_{p}\left(h_{n}(P)\right) \geq \tau_{\lambda}^{p}(n)$.

Proof. The theorem is an immediate consequence of Propositions 5.1 and 5.2.

\section{$6 \quad$ Abelian $p$-groups}

In this section we will provide two lemmas and go on to prove a key result (cf. Theorem 6.1) to Theorem 1.1.

Lemma 6.1 Suppose that $\lambda_{3} \geq 1$ and that $\sigma \in \Gamma_{n}$. Put $n_{0}=\sigma(0,0,1)$, $n_{1,2}=\sigma(1,2,1)$, and $n_{v, u}=\sigma(v, u, 1)$.

(1) Assume that either $u=\lambda_{1}$ or $u=v$. Excepting the case where $p=2$ and $u=v$, if $\left[n_{0} / p^{u+1}\right]<\left[n / p^{u+1}\right]$, then $(u-v+1) \gamma_{u+1, u}^{\sigma}(n)+\rho_{u}^{\sigma}(n) \geq u-v+1$.

(2) Assume that $u>\lambda_{1}$ and that $u=v+1$. If $\left[n_{0} / p^{u+1}\right]+\left[n_{v, u} / p\right]<\left[n / p^{u+1}\right]$, then $2 \gamma_{u+1, u}^{\sigma}(n)+\rho_{u}^{\sigma}(n) \geq 2$.

(3) Assume that $p=2$ and that $u=v$. If $\left[n_{0} / 2^{u+1}\right]+\left[n_{1,2} / 2^{u-1}\right]<\left[n / 2^{u+1}\right]$, then $\gamma_{u+1, u+1}^{\sigma}(n)+\rho_{u+1}^{\sigma}(n) \geq 1$. If $\left[n_{0} / 2^{u+3}\right]+\left[n_{1,2} / 2^{u+1}\right]<\left[n / 2^{u+3}\right]$, then $2 \gamma_{u+3, u+1}^{\sigma}(n)+\rho_{u+1}^{\sigma}(n) \geq 2$. 
Proof. (1) Since either $u=\lambda_{1}$ or $u=v$, it follows from Lemmas 4.1 and 4.2 that $c_{v, u}=0$. Thus $(v, u) \notin \widehat{\Omega}_{u}$. Hence, excepting the case where $p=2$ and $u=v$, if $\sigma(l, m, i) \geq p^{u-m+1}$ for some element $(l, m, i)$ of $I$ with $(l, m) \in \widehat{\Omega}_{u}$, then $(l, m) \neq(v, u)$ and $\rho_{u}^{\sigma}(n) \geq \kappa_{l, m}(\sigma(l, m, i)) \geq u-v+1$ by Lemma 5.2 and (1) of Lemma 5.3. Furthermore, if $\left[n_{0} / p^{u+1}\right]<\left[n / p^{u+1}\right]$ and if $\sigma(l, m, i)<p^{u-m+1}$ for any element $(l, m, i)$ of $I$ with $(l, m) \in \widehat{\Omega}_{u}$, then

$$
\gamma_{u+1, u}^{\sigma}(n)=\left[\frac{n}{p^{u+1}}\right]-\left[\frac{n_{0}}{p^{u+1}}\right]-\sum_{(l, m) \in \widehat{\Omega}_{u}} \sum_{i=1}^{c_{l, m}}\left[\frac{\sigma(l, m, i)}{p^{u-m+1}}\right] \geq 1 .
$$

Hence (1) follows.

(2) The hypothesis and Lemma 4.2 yield $c_{v, u}=1$; however, the proof is similar to that of (1) as follows. If $\sigma(l, m, i) \geq p^{u-m+1}$ for some element $(l, m, i)$ of $I$ with $(l, m) \in \widehat{\Omega}_{u}-\{(v, u)\}$, then $\rho_{u}^{\sigma}(n) \geq \kappa_{l, m}(\sigma(l, m, i)) \geq 2$ by Lemma 5.2 and (1) of Lemma 5.3. Moreover, if $\left[n_{0} / p^{u+1}\right]+\left[n_{v, u} / p\right]<\left[n / p^{u+1}\right]$ and if $\sigma(l, m, i)<p^{u-m+1}$ for any element $(l, m, i)$ of $I$ with $(l, m) \in \widehat{\Omega}_{u}-\{(v, u)\}$, then $\gamma_{u+1, u}^{\sigma}(n) \geq 1$. Now we get (2).

(3) Using Lemma 5.2 and (2) of Lemma 5.3, we can get the results. The proof is completely analogous to that of (2). Note that $c_{1,2}=1$ by the hypothesis and Lemma 4.1.

Lemma 6.2 Suppose that $P$ is a finite abelian p-group of type $\lambda$. Let $\sigma \in \Gamma_{n}$, and let $\Delta$ be a subset of $\Omega_{v}$. Then

$$
\operatorname{ord}_{p}\left(\frac{h_{n}^{\sigma}(P)}{\prod_{(l, m) \in \Delta} h_{\sigma(l, m, 1)}\left(C_{p^{u-l}} \times C_{p^{v-m}}\right)}\right) \geq \tau_{\lambda}^{p}(n)-\sum_{(l, m) \in \Delta} \tau_{\lambda}^{p, m}(\sigma(l, m, 1)) .
$$

Proof. The lemma follows from Lemmas 5.1, 5.2, and 5.3.

Theorem 6.1 Suppose that $P$ is a finite abelian p-group of type $\lambda$. Let $y_{n}=$ $\left[n / p^{u+1}\right]$ for each nonnegative integer $n$. Put $\delta=1$ if either $p>2$ or $s=u$, and is 0 otherwise.

(1) Excepting the case where $p=2$ and $2 u=s \geq 2$, if either $u=\lambda_{1}$ or $2 u=s$, then

$$
h_{n}(P) \equiv \frac{(-1)^{y_{n}} n !}{p^{(2 u-s+1) y_{n}} y_{n} !\left(n-p^{u+1} y_{n}\right) !} h_{n-p^{u+1} y_{n}}(P) \quad \bmod p^{\tau_{\lambda}^{p}(n)+2 u-s+\delta} .
$$


(2) If $u>\lambda_{1}$ and if $2 u=s+1$, then

$$
h_{n}(P) \equiv \frac{(-1-p)^{y_{n}} n !}{p^{2 y_{n}} y_{n} !\left(n-p^{u+1} y_{n}\right) !} h_{n-p^{u+1} y_{n}}(P) \quad \bmod p^{\tau_{\lambda}^{p}(n)+1+\delta} .
$$

Proof. (1) If $\lambda_{3}=0$, then the assertion follows from Theorem 2.1. Hence we assume that $\lambda_{3} \geq 1$. Assume that either $p>2$ or $u>v$ and that either $u=\lambda_{1}$ or $u=v$. Set $\widehat{\Omega}=\Omega-\{(0,0)\}$ and

$$
\widehat{\Gamma}_{n}=\left\{\sigma \in \Gamma_{n} \mid\left[\sigma(0,0,1) / p^{u+1}\right]=y_{n}\right\} .
$$

Then by Proposition 5.1, Lemma 5.1, and (1) of Lemma 6.1,

$$
h_{n}(P) \equiv \sum_{\sigma \in \widehat{\Gamma}_{n}} h_{n}^{\sigma}(P) \quad \bmod p^{\tau_{\lambda}^{p}(n)+u-v+1} .
$$

Suppose that $\sigma \in \widehat{\Gamma}_{n}$. Since $c_{0,0}=1$, it follows that

$$
h_{n}^{\sigma}(P)=n ! \frac{h_{n_{0}^{\sigma}}\left(C_{p^{u}} \times C_{p^{v}}\right)}{n_{0}^{\sigma} !} \prod_{(l, m) \in \widehat{\Omega}} \prod_{i=1}^{c_{l, m}} \frac{g_{l, m}(\sigma(l, m, i))}{\sigma(l, m, i) !}
$$

where $n_{0}^{\sigma}=\sigma(0,0,1)$. Moreover, using Theorem 2.1, we obtain $h_{n_{0}^{\sigma}}\left(C_{p^{u}} \times C_{p^{v}}\right) \equiv \frac{(-1)^{y_{n}} n_{0}^{\sigma} !}{p^{(u-v+1) y_{n}} y_{n} !} \cdot \frac{h_{n_{0}^{\sigma}-p^{u+1} y_{n}}\left(C_{p^{u}} \times C_{p^{v}}\right)}{\left(n_{0}^{\sigma}-p^{u+1} y_{n}\right) !} \bmod p^{\tau_{\lambda}^{p}\left(n_{0}^{\sigma}\right)+u-v+\delta}$.

Hence Lemma 6.2 with $\Delta=\{(0,0)\}$ implies that

$$
\begin{array}{r}
h_{n}^{\sigma}(P) \equiv \frac{(-1)^{y_{n}} n !}{p^{(u-v+1) y_{n}} y_{n} !} \cdot \frac{h_{n_{0}^{\sigma}-p^{u+1} y_{n}}\left(C_{p^{u}} \times C_{p^{v}}\right)}{\left(n_{0}^{\sigma}-p^{u+1} y_{n}\right) !} \prod_{(l, m) \in \widehat{\Omega}} \prod_{i=1}^{c_{l, m}} \frac{g_{l, m}(\sigma(l, m, i))}{\sigma(l, m, i) !} \\
\bmod p^{\tau_{\lambda}^{p}(n)+u-v+\delta} .
\end{array}
$$

Now, combining the formula above with (I), we conclude that

$$
\begin{aligned}
h_{n}(P) & \equiv \frac{(-1)^{y_{n}} n !}{p^{(u-v+1) y_{n}} y_{n} !} \sum_{\sigma \in \widehat{\Gamma}_{n}} \frac{h_{n_{0}^{\sigma}-p^{u+1} y_{n}}\left(C_{p^{u}} \times C_{p^{v}}\right)}{\left(n_{0}^{\sigma}-p^{u+1} y_{n}\right) !} \prod_{(l, m) \in \widehat{\Omega}} \prod_{i=1}^{c_{l, m}} \frac{g_{l, m}(\sigma(l, m, i))}{\sigma(l, m, i) !} \\
& \equiv \frac{(-1)^{y_{n}} n !}{p^{(u-v+1) y_{n}} y_{n} !} \sum_{\sigma \in \Gamma_{n-p^{u+1} y_{n}}} \prod_{(l, m) \in \Omega} \prod_{i=1}^{c_{l, m}} \frac{g_{l, m}(\sigma(l, m, i))}{\sigma(l, m, i) !} \\
& \equiv \frac{(-1)^{y_{n}} n !}{p^{(u-v+1) y_{n}} y_{n} !\left(n-p^{u+1} y_{n}\right) !} h_{n-p^{u+1} y_{n}}(P) \bmod p^{\tau_{\lambda}^{p}(n)+u-v+\delta} .
\end{aligned}
$$


Here Proposition 5.1 is applied to the last congruence. Thus (1) follows.

(2) Assume that $u>\lambda_{1}$ and that $u=v+1$. By Lemma 4.2, we have $c_{v, u}=1$. Set $\widetilde{\Omega}=\Omega-\{(0,0),(v, u)\}$ and

$$
\widetilde{\Gamma}_{n}(y)=\left\{\sigma \in \Gamma_{n} \mid[\sigma(v, u, 1) / p]=y,\left[\sigma(0,0,1) / p^{u+1}\right]=y_{n}-y\right\}
$$

for each integer $y$ with $0 \leq y \leq y_{n}$. Then by Proposition 5.1, Lemma 5.1, and (2) of Lemma 6.1,

$$
h_{n}(P) \equiv \sum_{y=0}^{y_{n}} \sum_{\sigma \in \widetilde{\Gamma}_{n}(y)} h_{n}^{\sigma}(P) \quad \bmod p^{\tau_{\lambda}^{p}(n)+2} .
$$

Suppose that $\sigma \in \widetilde{\Gamma}_{n}(y)$. Since $c_{0,0}=c_{v, u}=1$, it follows that

$$
h_{n}^{\sigma}(P)=n ! \frac{h_{n_{0}^{\sigma}}\left(C_{p^{u}} \times C_{p^{v}}\right)}{n_{0}^{\sigma} !} \cdot \frac{1}{n_{v, u}^{\sigma} !} \prod_{(l, m) \in \widetilde{\Omega}} \prod_{i=1}^{c_{l, m}} \frac{g_{l, m}(\sigma(l, m, i))}{\sigma(l, m, i) !},
$$

where $n_{0}^{\sigma}=\sigma(0,0,1)$ and $n_{v, u}^{\sigma}=\sigma(v, u, 1)$. Observe now that by Lemmas 5.1, 5.2 , and 5.3,

$$
\operatorname{ord}_{p}\left(h_{n}^{\sigma}(P)\right)-\tau_{\lambda}^{p}(n) \geq \rho_{u}^{\sigma}(n) \geq \kappa_{v, u}\left(n_{v, u}^{\sigma}\right)=y .
$$

By Theorem 2.1 with $P$ is the group consisting of only the identity, we also have

$$
1 \equiv \frac{(-1)^{y} n_{v, u}^{\sigma} !}{p^{y} y !\left(n_{v, u}^{\sigma}-p y\right) !} \bmod p .
$$

Hence the preceding formula of $h_{n}^{\sigma}(P)$ yields

$$
\begin{array}{r}
h_{n}^{\sigma}(P) \equiv n ! \frac{h_{n_{0}^{\sigma}}\left(C_{p^{u}} \times C_{p^{v}}\right)}{n_{0}^{\sigma} !} \cdot \frac{(-1)^{y}}{p^{y} y !\left(n_{v, u}^{\sigma}-p y\right) !} \prod_{(l, m) \in \widetilde{\Omega}} \prod_{i=1}^{c_{l, m}} \frac{g_{l, m}(\sigma(l, m, i))}{\sigma(l, m, i) !} \\
\bmod p^{\tau_{\lambda}^{p}(n)+2},
\end{array}
$$

and also, Lemma 6.2 with $\Delta=\{(0,0)\}$ implies that

$$
\begin{aligned}
\operatorname{ord}_{p}\left(\frac{n !}{n_{0}^{\sigma} !} \cdot \frac{(-1)^{y}}{p^{y} y !\left(n_{v, u}^{\sigma}-p y\right) !} \prod_{(l, m) \in \widetilde{\Omega}} \prod_{i=1}^{c_{l, m}} \frac{g_{l, m}(\sigma(l, m, i))}{\sigma(l, m, i) !}\right) \\
\quad=\operatorname{ord}_{p}\left(\frac{h_{n}^{\sigma}(P)}{h_{n_{0}^{\sigma}}\left(C_{p^{u}} \times C_{p^{v}}\right)} \cdot \frac{(-1)^{y} n_{v, u}^{\sigma} !}{p^{y} y !\left(n_{v, u}^{\sigma}-p y\right) !}\right) \geq \tau_{\lambda}^{p}(n)-\tau_{\lambda}^{p, 0}\left(n_{0}^{\sigma}\right) .
\end{aligned}
$$


(Note that the congruence above is clear if $y=0$.) Moreover,

$$
\begin{aligned}
h_{n_{0}^{\sigma}}\left(C_{p^{u}} \times\right. & \left.C_{p^{v}}\right) \\
& \equiv \frac{(-1)^{y_{n}-y} n_{0}^{\sigma} !}{p^{2\left(y_{n}-y\right)}\left(y_{n}-y\right) !} \cdot \frac{h_{n_{0}^{\sigma}-p^{u+1}\left(y_{n}-y\right)}\left(C_{p^{u}} \times C_{p^{v}}\right)}{\left(n_{0}^{\sigma}-p^{u+1}\left(y_{n}-y\right)\right) !} \bmod p^{\tau_{\lambda}^{p}\left(n_{0}^{\sigma}\right)+1+\delta}
\end{aligned}
$$

by Theorem 2.1, and consequently,

$$
\begin{aligned}
h_{n}^{\sigma}(P) \equiv \frac{(-1)^{y_{n}} n !}{p^{2 y_{n}-y}\left(y_{n}-y\right) ! y !} & \frac{h_{n_{0}^{\sigma}-p^{u+1}\left(y_{n}-y\right)}\left(C_{p^{u}} \times C_{p^{v}}\right)}{\left(n_{0}^{\sigma}-p^{u+1}\left(y_{n}-y\right)\right) !} \cdot \frac{1}{\left(n_{v, u}^{\sigma}-p y\right) !} \\
& \times \prod_{(l, m) \in \widetilde{\Omega}} \prod_{i=1}^{c_{l, m}} \frac{g_{l, m}(\sigma(l, m, i))}{\sigma(l, m, i) !} \bmod p^{\tau_{\lambda}^{p}(n)+1+\delta} .
\end{aligned}
$$

Now, the formula above, together with $(\mathrm{J})$, yields

$$
\begin{array}{r}
h_{n}(P) \equiv \sum_{y=0}^{y_{n}} \frac{(-1)^{y_{n}} n !}{p^{2 y_{n}-y}\left(y_{n}-y\right) ! y !} \sum_{\sigma \in \widetilde{\Gamma}_{n}(y)} \frac{h_{n_{0}^{\sigma}-p^{u+1}\left(y_{n}-y\right)}\left(C_{p^{u}} \times C_{p^{v}}\right)}{\left(n_{0}^{\sigma}-p^{u+1}\left(y_{n}-y\right)\right) !} \cdot \frac{1}{\left(n_{v, u}^{\sigma}-p y\right) !} \\
\times \prod_{(l, m) \in \widetilde{\Omega}_{i=1}}^{c_{l, m}} \frac{g_{l, m}(\sigma(l, m, i))}{\sigma(l, m, i) !} \\
\equiv \frac{(-1)^{y_{n}} n !}{p^{2 y_{n}}} \sum_{y=0}^{y_{n}} \frac{p^{y}}{\left(y_{n}-y\right) ! y !} \sum_{\sigma \in \Gamma_{n-p^{u+1} y_{n}}} \prod_{(l, m) \in \Omega} \prod_{i=1}^{c_{l, m}} \frac{g_{l, m}(\sigma(l, m, i))}{\sigma(l, m, i) !} \\
\bmod p^{\tau_{\lambda}^{p}(n)+1+\delta .} .
\end{array}
$$

Hence it follows from Proposition 5.1 that

$$
\begin{aligned}
h_{n}(P) & \equiv \frac{(-1)^{y_{n}} n !}{p^{2 y_{n}} y_{n} !}\left\{\sum_{y=0}^{y_{n}} \frac{y_{n} ! p^{y}}{\left(y_{n}-y\right) ! y !}\right\} \frac{h_{n-p^{u+1} y_{n}}(P)}{\left(n-p^{u+1} y_{n}\right) !} \\
& \equiv \frac{(-1-p)^{y_{n}} n !}{p^{2 y_{n}} y_{n} !\left(n-p^{u+1} y_{n}\right) !} h_{n-p^{u+1} y_{n}}(P) \bmod p^{\tau_{\lambda}^{p}(n)+1+\delta} .
\end{aligned}
$$

Thus (2) follows. This completes the proof.

\section{Abelian 2-groups}

Throughout this section we suppose that $p=2$. If $2 u=s \geq 2$ as well, then the properties of $h_{n}(P)$, where $P$ is a finite abelian 2-group of type $\lambda$, are a little complicated (cf. Theorems 7.1 and 7.2). First, we provide the following. 
Lemma 7.1 Suppose that $u \geq 2$. Put $w=\left[n / 2^{u}\right]$ and $z=\left[n / 2^{u+1}\right]$. Then

$$
h_{n}\left(C_{2^{u-1}} \times C_{2^{u-2}}\right) \equiv \frac{n !}{2^{5 z} z !} \cdot \frac{h_{n-2^{u+1} z}\left(C_{2^{u-1}} \times C_{2^{u-2}}\right)}{\left(n-2^{u+1} z\right) !} \bmod 2^{f_{2}^{u-1}(n)-w+1} .
$$

Proof. Put $n^{\prime}=n-2^{u+1} z$ and $w^{\prime}=w-2 z$. Then by Theorem 2.1,

$h_{n^{\prime}}\left(C_{2^{u-1}} \times C_{2^{u-2}}\right) \equiv \frac{(-1)^{w^{\prime}} n^{\prime} !}{2^{2 w^{\prime}} w^{\prime} !} \cdot \frac{h_{n-2^{u} w}\left(C_{2^{u-1}} \times C_{2^{u-2}}\right)}{\left(n-2^{u} w\right) !} \bmod 2^{f_{2}^{u-1}\left(n^{\prime}\right)-w^{\prime}+1}$.

Since $z=[w / 2]$, it follows from Theorem 2.1 that

$$
1 \equiv \frac{w !}{2^{z} z ! w^{\prime} !} \bmod 2 .
$$

Hence it follows from Theorem 2.1 that

$$
\begin{aligned}
h_{n}\left(C_{2^{u-1}} \times C_{2^{u-2}}\right) & \equiv \frac{(-1)^{w} n !}{2^{2 w} w !} \cdot \frac{h_{n-2^{u} w}\left(C_{2^{u-1}} \times C_{2^{u-2}}\right)}{\left(n-2^{u} w\right) !} \\
& \equiv \frac{(-1)^{w} n !}{2^{2 w} w !} \cdot \frac{w !}{2^{z} z ! w^{\prime} !} \cdot \frac{h_{n-2^{u} w}\left(C_{2^{u-1}} \times C_{2^{u-2}}\right)}{\left(n-2^{u} w\right) !} \\
& \equiv \frac{n !}{2^{5 z} z ! n^{\prime} !} \cdot \frac{(-1)^{w^{\prime}} n^{\prime} !}{2^{2 w^{\prime}} w^{\prime} !} \cdot \frac{h_{n-2^{u} w}\left(C_{2^{u-1}} \times C_{2^{u-2}}\right)}{\left(n-2^{u} w\right) !} \\
& \equiv \frac{n !}{2^{5 z} z !} \cdot \frac{h_{n^{\prime}}\left(C_{2^{u-1}} \times C_{2^{u-2}}\right)}{n^{\prime} !} \bmod 2^{f_{2}^{u-1}(n)-w+1}
\end{aligned}
$$

completing the proof.

We correct the second assertions of [9, Theorem 4.2] and [18, Theorem 2.1(b)] with $l \geq 2$, together with [18, Theorem 3.10(d)].

Theorem 7.1 Suppose that $2 u=s \geq 2$ and that $P$ is a finite abelian 2-group of type $\lambda$. Let $z_{n}=\left[n / 2^{u+3}\right]$ for each nonnegative integer $n$.

(1) If $\lambda_{3}=0$ and if $u \geq 2$, then

$$
h_{n}(P) \equiv \frac{n !}{2^{6 z_{n}} z_{n} !\left(n-2^{u+3} z_{n}\right) !} h_{n-2^{u+3} z_{n}}(P) \bmod 2^{\tau_{\lambda}^{2}(n)+2} .
$$

(2) If either $\lambda_{3} \geq 1$ or $u=1$, then

$$
h_{n}(P) \equiv \frac{(-1)^{z_{n}} n !}{2^{6 z_{n}} z_{n} !\left(n-2^{u+3} z_{n}\right) !} h_{n-2^{u+3} z_{n}}(P) \quad \bmod 2^{\tau_{\lambda}^{2}(n)+2} .
$$


Proof. (1) Suppose that $\lambda=(u, u, 0, \ldots)$ with $u \geq 2$. We argue by induction on $u$. Let $Q=C_{2^{u-1}} \times C_{2^{u-1}}$, and set $n^{\prime}=n-2^{u+3} z_{n}$. We use the formula

$$
h_{n}(P)=\sum_{i=0}^{[n / 2]} \frac{n !}{(n-2 i) ! i !} h_{n-2 i}\left(C_{2^{2 u}}\right) h_{i}(Q)
$$

(see [9]). Since $2^{u} \geq 2 u$, it follows that for any nonnegative integers $k, l$, and $m$ such that $k<2^{u+1}$ and $l<2^{u}$,

$$
\left[\frac{k+2^{u+1} l+2^{2 u+1} m}{2^{u+1}}\right]-2 u\left[\frac{k+2^{u+1} l+2^{2 u+1} m}{2^{2 u+1}}\right]=l+\left(2^{2 u}-2 u\right) m \geq 0 .
$$

This, combined with Theorem 5.1, shows that

$$
\begin{aligned}
\operatorname{ord}_{2}( & \left.\frac{n !}{i !(n-2 i) !} h_{n-2 i}\left(C_{2^{2 u}}\right) h_{i}(Q)\right) \\
\geq & \sum_{j=1}^{\infty}\left[\frac{n}{2^{j}}\right]-\sum_{j=1}^{\infty}\left[\frac{i}{2^{j}}\right]-\sum_{j=1}^{\infty}\left[\frac{n-2 i}{2^{j}}\right]+\sum_{j=1}^{2 u}\left[\frac{n-2 i}{2^{j}}\right]-2 u\left[\frac{n-2 i}{2^{2 u+1}}\right] \\
& +\sum_{j=1}^{u-1}\left[\frac{i}{2^{j}}\right]+\left[\frac{i}{2^{u+1}}\right]-\left[\frac{i}{2^{u+2}}\right] \\
\geq & \sum_{j=1}^{u}\left[\frac{n}{2^{j}}\right]+\left[\frac{n}{2^{u+2}}\right]-\left[\frac{n}{2^{u+3}}\right]+\left(\left[\frac{n}{2^{u+1}}\right]-\left[\frac{2 i}{2^{u+1}}\right]-\left[\frac{n-2 i}{2^{u+1}}\right]\right) \\
& +\sum_{j=u+3}^{\infty}\left(\left[\frac{n}{2^{j}}\right]-\left[\frac{2 i}{2^{j}}\right]-\left[\frac{n-2 i}{2^{j}}\right]\right)+\sum_{j=u+3}^{2 u}\left[\frac{n-2 i}{2^{j}}\right]+\left[\frac{n}{2^{u+3}}\right]-\left[\frac{i}{2^{u+2}}\right] \\
\geq & \tau_{\lambda}^{2}(n)+2\left(\left[\frac{n}{2^{u+3}}\right]-\left[\frac{2 i}{2^{u+3}}\right]-\left[\frac{n-2 i}{2^{u+3}}\right]\right)+\left[\frac{n-2 i}{2^{u+3}}\right]+\sum_{j=u+3}^{2 u}\left[\frac{n-2 i}{2^{j}}\right] .
\end{aligned}
$$

(When $u=2$, the corresponding part in the proof of [9, Theorem 4.2] is not correct.) Suppose that $u=2$. If $n-2^{5}\left(z_{n}-z+1\right)<n-2 i<2^{5} z$ with $z=1,2, \ldots z_{n}$, then

$$
\operatorname{ord}_{2}\left(\frac{n !}{(n-2 i) ! i !} h_{n-2 i}\left(C_{2^{4}}\right) h_{i}(Q)\right) \geq \tau_{\lambda}^{2}(n)+2
$$

Hence

$$
h_{n}(P) \equiv \sum_{z=0}^{z_{n}} \sum_{i=2^{4} z}^{[n / 2]-2^{4}\left(z_{n}-z\right)} \frac{n !}{(n-2 i) ! i !} h_{n-2 i}\left(C_{2^{4}}\right) h_{i}(Q) \bmod 2^{\tau_{\lambda}^{2}(n)+2} .
$$


Observe now that by $[9$, Theorem 4.1],

$$
h_{i}(Q) \equiv \frac{(-1)^{z} i !}{2^{6 z} z !\left(i-2^{4} z\right) !} h_{i-2^{4} z}(Q) \quad \bmod 2^{[i / 2]+\left[i / 2^{3}\right]-\left[i / 2^{4}\right]+2},
$$

where $0 \leq z \leq z_{n}$ and $2^{4} z \leq i \leq[n / 2]-2^{4}\left(z_{n}-z\right)$. Then we obtain

$$
\begin{aligned}
h_{n}(P) & \equiv \sum_{z=0}^{z_{n}} \frac{(-1)^{z} n !}{2^{6 z} z !} \sum_{i=2^{4} z}^{[n / 2]-2^{4}\left(z_{n}-z\right)} \frac{h_{n-2 i}\left(C_{2^{4}}\right)}{(n-2 i) !} \cdot \frac{h_{i-2^{4} z}(Q)}{\left(i-2^{4} z\right) !} \\
& \equiv \sum_{z=0}^{z_{n}} \frac{(-1)^{z} n !}{2^{6 z} z !} \sum_{i=0}^{\left[n^{\prime} / 2\right]} \frac{h_{n-2 i-2^{5} z}\left(C_{2^{4}}\right)}{\left(n-2 i-2^{5} z\right) !} \cdot \frac{h_{i}(Q)}{i !} \bmod 2^{\tau_{\lambda}^{2}(n)+2} .
\end{aligned}
$$

Moreover, it follows from Theorem 5.1 that

$$
\begin{aligned}
h_{n}(P) & \equiv \sum_{z=0}^{z_{n}} \frac{(-1)^{z_{n}} n !}{2^{5 z_{n}+z}\left(z_{n}-z\right) ! z !} \sum_{i=0}^{\left[n^{\prime} / 2\right]} \frac{h_{n^{\prime}-2 i}\left(C_{2^{4}}\right)}{\left(n^{\prime}-2 i\right) !} \cdot \frac{h_{i}(Q)}{i !} \\
& \equiv \frac{(-3)^{z_{n}} n !}{2^{6 z_{n}} z_{n} !} \sum_{i=0}^{\left[n^{\prime} / 2\right]} \frac{h_{n^{\prime}-2 i}\left(C_{2^{4}}\right)}{\left(n^{\prime}-2 i\right) !} \cdot \frac{h_{i}(Q)}{i !} \\
& \equiv \frac{n !}{2^{6 z_{n}} z_{n} ! n^{\prime} !} h_{n^{\prime}}(P) \bmod 2^{\tau_{\lambda}^{2}(n)+2},
\end{aligned}
$$

as desired. Suppose next that $u \geq 3$. If $n-2^{u+3} z_{n}<n-2 i$, then

$$
\begin{aligned}
& \operatorname{ord}_{2}\left(\frac{n !}{(n-2 i) ! i !} h_{n-2 i}\left(C_{2^{2 u}}\right) h_{i}(Q)\right) \\
& \geq \tau_{\lambda}^{2}(n)+2\left(\left[\frac{n}{2^{u+3}}\right]-\left[\frac{2 i}{2^{u+3}}\right]-\left[\frac{n-2 i}{2^{u+3}}\right]\right)+\left[\frac{n-2 i}{2^{u+3}}\right]+\sum_{j=u+3}^{2 u}\left[\frac{n-2 i}{2^{j}}\right] \\
& \quad \geq \tau_{\lambda}^{2}(n)+2 .
\end{aligned}
$$

Thus

$$
h_{n}(P) \equiv \sum_{i=2^{u+2} z_{n}}^{[n / 2]} \frac{n !}{(n-2 i) ! i !} h_{n-2 i}\left(C_{2^{2 u}}\right) h_{i}(Q) \bmod 2^{\tau_{\lambda}^{2}(n)+2} .
$$

By the inductive assumption,

$$
h_{i}(Q) \equiv \frac{i !}{2^{6 z_{n}} z_{n} !\left(i-2^{u+2} z_{n}\right) !} h_{i-2^{u+2} z_{n}}(Q) \quad \bmod 2^{f_{2}^{u-1}(i)+\left[i / 2^{u+1}\right]-z_{n}+2},
$$


where $2^{u+2} z_{n} \leq i \leq[n / 2]$. We now obtain

$$
\begin{aligned}
h_{n}(P) & \equiv \frac{n !}{2^{6 z_{n}} z_{n} !} \sum_{i=2^{u+2} z_{n}}^{[n / 2]} \frac{h_{n-2 i}\left(C_{2^{2 u}}\right)}{(n-2 i) !} \cdot \frac{h_{i-2^{u+2} z_{n}}(Q)}{\left(i-2^{u+2} z_{n}\right) !} \\
& \equiv \frac{n !}{2^{6 z_{n}} z_{n} !} \sum_{i=0}^{\left[n^{\prime} / 2\right]} \frac{h_{n^{\prime}-2 i}\left(C_{2^{2 u}}\right)}{\left(n^{\prime}-2 i\right) !} \cdot \frac{h_{i}(Q)}{i !} \\
& \equiv \frac{n !}{2^{6 z_{n}} z_{n} ! n^{\prime} !} h_{n^{\prime}}(P) \bmod 2^{\tau_{\lambda}^{2}(n)+2},
\end{aligned}
$$

as desired. Thus (1) holds.

(2) If $u=1$, then the assertion is stated in [9, Theorem 4.1], and is already proved. Suppose that $u \geq 2$ and that $\lambda_{3} \geq 1$. Then, since $u=v \geq 2$, it follows from Lemma 4.1 that $c_{1,2}=1$. Set

$$
\bar{\Omega}=\Omega-\{(0,0),(1,2)\}
$$

and

$$
\bar{\Gamma}_{n}(z)=\left\{\sigma \in \Gamma_{n} \mid\left[\sigma(1,2,1) / 2^{u+1}\right]=z,\left[\sigma(0,0,1) / 2^{u+3}\right]=z_{n}-z\right\}
$$

for each integer $z$ with $0 \leq z \leq z_{n}$. Then by Proposition 5.1, Lemma 5.1, and (3) of Lemma 6.1,

$$
h_{n}(P) \equiv \sum_{z=0}^{z_{n}} \sum_{\sigma \in \bar{\Gamma}_{n}(z)} h_{n}^{\sigma}(P) \quad \bmod 2^{\tau_{\lambda}^{2}(n)+2} .
$$

Since $c_{0,0}=c_{1,2}=1$, it follows that for any $\sigma \in \Gamma_{n}$,

$$
\begin{aligned}
h_{n}^{\sigma}(P)=n ! \frac{h_{n_{0}^{\sigma}}\left(C_{2^{u}} \times C_{2^{u}}\right)}{n_{0}^{\sigma} !} \cdot \frac{h_{n_{1,2}^{\sigma}}\left(C_{2^{u-1}} \times C_{2^{u-2}}\right)}{n_{1,2}^{\sigma} !} \\
\times \prod_{(l, m) \in \bar{\Omega}} \prod_{i=1}^{c_{l, m}} \frac{g_{l, m}(\sigma(l, m, i))}{\sigma(l, m, i) !},
\end{aligned}
$$

where $n_{0}^{\sigma}=\sigma(0,0,1)$ and $n_{1,2}^{\sigma}=\sigma(1,2,1)$.

Suppose that $\sigma \in \bar{\Gamma}_{n}(z)$. Then Lemma 7.1 implies that

$$
\begin{aligned}
h_{n_{1,2}^{\sigma}}\left(C_{2^{u-1}}\right. & \left.\times C_{2^{u-2}}\right) \\
& \equiv \frac{n_{1,2}^{\sigma} !}{2^{5 z} z !} \cdot \frac{h_{n_{1,2}^{\sigma}-2^{u+1} z}\left(C_{2^{u-1}} \times C_{2^{u-2}}\right)}{\left(n_{1,2}^{\sigma}-2^{u+1} z\right) !} \bmod 2^{f_{2}^{u-1}\left(n_{1,2}^{\sigma}\right)-\left[n_{1,2}^{\sigma} / 2^{u}\right]+1} .
\end{aligned}
$$


Also, it follows from (1) that $h_{n_{0}^{\sigma}}\left(C_{2^{u}} \times C_{2^{u}}\right) \equiv \frac{n_{0}^{\sigma} !}{2^{6\left(z_{n}-z\right)}\left(z_{n}-z\right) !} \cdot \frac{h_{n_{0}^{\sigma}-2^{u+3}\left(z_{n}-z\right)}\left(C_{2^{u}} \times C_{2^{u}}\right)}{\left(n_{0}^{\sigma}-2^{u+3}\left(z_{n}-z\right)\right) !} \bmod 2^{\tau_{\lambda}^{2}\left(n_{0}^{\sigma}\right)+2}$.

Hence (L), combined with Theorem 5.1, yields

$$
\begin{aligned}
h_{n}^{\sigma}(P) \equiv & \frac{n !}{2^{6 z_{n}-z}\left(z_{n}-z\right) ! z !} \cdot \frac{h_{n_{0}^{\sigma}-2^{u+3}\left(z_{n}-z\right)}\left(C_{2^{u}} \times C_{2^{u}}\right)}{\left(n_{0}^{\sigma}-2^{u+3}\left(z_{n}-z\right)\right) !} \\
& \times \frac{h_{n_{1,2}^{\sigma}-2^{u+1} z}\left(C_{2^{u-1}} \times C_{2^{u-2}}\right)}{\left(n_{1,2}^{\sigma}-2^{u+1} z\right) !} \prod_{(l, m) \in \bar{\Omega}} \prod_{i=1}^{c_{l, m}} \frac{g_{l, m}(\sigma(l, m, i))}{\sigma(l, m, i) !} \bmod 2^{\tau_{\lambda}^{2}(n)+2},
\end{aligned}
$$

because

$$
\operatorname{ord}_{2}\left(\frac{h_{n}^{\sigma}(P)}{h_{n_{0}^{\sigma}}\left(C_{2^{u}} \times C_{2^{u}}\right) h_{n_{1,2}^{\sigma}}\left(C_{2^{u-1}} \times C_{2^{u-2}}\right)}\right) \geq \tau_{\lambda}^{2}(n)-\tau_{\lambda}^{2,0}\left(n_{0}^{\sigma}\right)-\tau_{\lambda}^{2,2}\left(n_{1,2}^{\sigma}\right)
$$

by Lemma 6.2 with $\Delta=\{(0,0),(1,2)\}$ and

$$
f_{2}^{u-1}\left(n_{1,2}^{\sigma}\right)-\left[\frac{n_{1,2}^{\sigma}}{2^{u}}\right]-\tau_{\lambda}^{2,2}\left(n_{1,2}^{\sigma}\right)=\left[\frac{n_{1,2}^{\sigma}}{2^{u-1}}\right]-2\left[\frac{n_{1,2}^{\sigma}}{2^{u}}\right]+z \geq z .
$$

Now, combining the preceding formula of $h_{n}^{\sigma}(P)$ with $(\mathrm{K})$, we have

$$
\begin{array}{r}
h_{n}(P) \equiv \sum_{z=0}^{z_{n}} \frac{n !}{2^{6 z_{n}-z}\left(z_{n}-z\right) ! z !} \sum_{\sigma \in \bar{\Gamma}_{n}(z)} \frac{h_{n_{0}^{\sigma}-2^{u+3}\left(z_{n}-z\right)}\left(C_{2^{u}} \times C_{2^{u}}\right)}{\left(n_{0}^{\sigma}-2^{u+3}\left(z_{n}-z\right)\right) !} \\
\times \frac{h_{n_{1,2}^{\sigma}-2^{u+1} z}\left(C_{2^{u-1}} \times C_{2^{u-2}}\right)}{\left(n_{1,2}^{\sigma}-2^{u+1} z\right) !} \prod_{(l, m) \in \bar{\Omega}} \prod_{i=1}^{c_{l, m}} \frac{g_{l, m}(\sigma(l, m, i))}{\sigma(l, m, i) !} \\
\equiv \frac{n !}{2^{6 z_{n}}} \sum_{z=0}^{z_{n}} \frac{2^{z}}{\left(z_{n}-z\right) ! z !} \sum_{\sigma \in \Gamma_{n-2^{u+3} z_{n}}} \prod_{(l, m) \in \Omega} \prod_{i=1}^{c_{l, m}} \frac{g_{l, m}(\sigma(l, m, i))}{\sigma(l, m, i) !} \\
\bmod 2^{\tau_{\lambda}^{2}(n)+2} .
\end{array}
$$

Hence Proposition 5.1, together with Theorem 5.2, yields

$$
\begin{aligned}
h_{n}(P) & \equiv \frac{n !}{2^{6 z_{n}} z_{n} !}\left\{\sum_{z=0}^{z_{n}} \frac{2^{z} z_{n} !}{\left(z_{n}-z\right) ! z !}\right\} \frac{h_{n-2^{u+3} z_{n}}(P)}{\left(n-2^{u+3} z_{n}\right) !} \\
& \equiv \frac{(-1)^{z_{n}} n !}{2^{6 z_{n}} z_{n} !\left(n-2^{u+3} z_{n}\right) !} h_{n-2^{u+3} z_{n}}(P) \bmod 2^{\tau_{\lambda}^{2}(n)+2}
\end{aligned}
$$


as desired. This competes the proof.

The following theorem, as well as Theorem 7.1, includes the interesting difference arising from the value of $\lambda_{3}$.

Theorem 7.2 Suppose that $2 u=s \geq 2$ and that $P$ is a finite abelian 2-group of type $\lambda$. Let $y_{n}=\left[n / 2^{u+1}\right]$ for each nonnegative integer $n$.

(1) Assume that $\lambda_{3}=0$. Then

$$
h_{n}(P) \equiv \frac{n !}{2^{y_{n}} y_{n} !\left(n-2^{u+1} y_{n}\right) !} h_{y_{n}}\left(C_{2}\right) h_{n-2^{u+1} y_{n}}(P) \quad \bmod 2^{\tau_{\lambda}^{2}(n)+1} .
$$

In particular, if $y_{n} \equiv 3 \bmod 4$, then $h_{n}(P) \equiv 0 \bmod 2^{\tau_{\lambda}^{2}(n)+1}$.

(2) Assume that $\lambda_{3} \geq 1$.

(i) If either $y_{n} \equiv 0 \bmod 4$ or $y_{n} \equiv 1 \bmod 4$, then

$$
h_{n}(P) \equiv \frac{n !}{2^{y_{n}} y_{n} !\left(n-2^{u+1} y_{n}\right) !} h_{y_{n}}\left(C_{2}\right) h_{n-2^{u+1} y_{n}}(P) \quad \bmod 2^{\tau_{\lambda}^{2}(n)+1} .
$$

(ii) If $y_{n} \equiv 2 \bmod 4$, then $h_{n}(P) \equiv 0 \bmod 2^{\tau_{\lambda}^{2}(n)+1}$.

(iii) If $y_{n} \equiv 3 \bmod 4$, then

$$
\begin{aligned}
h_{n}(P) \equiv \frac{n !}{2^{y_{n}}\left(y_{n}-2\right) !\left(n-2^{u+1} y_{n}\right) !} h_{y_{n}-2}\left(C_{2}\right) h_{n-2^{u+1} y_{n}}(P) & \bmod 2^{\tau_{\lambda}^{2}(n)+1} .
\end{aligned}
$$

Proof. (1) The first assertion is a part of Theorem 2.2, and is already proved. It follows from [12] (see also $[4,9]$ ) that

$$
\operatorname{ord}_{2}\left(h_{n}\left(C_{2}\right)\right)= \begin{cases}{\left[\frac{n}{2}\right]-\left[\frac{n}{4}\right]} & \text { if } n \equiv 0,1,2 \bmod 4 \\ {\left[\frac{n}{2}\right]-\left[\frac{n}{4}\right]+1} & \text { if } \quad n \equiv 3 \quad \bmod 4\end{cases}
$$

Hence, if $\lambda_{3}=0$ and if $y_{n} \equiv 3 \bmod 4$, then by the congruence in (1) and Theorem 5.2,

$$
\begin{aligned}
\operatorname{ord}_{2}\left(h_{n}(P)\right) & \geq \operatorname{ord}_{2}(n !)-y_{n}-\operatorname{ord}_{2}\left(y_{n} !\right)+\operatorname{ord}_{2}\left(h_{y_{n}}\left(C_{2}\right)\right) \\
& =f_{2}^{u}(n)+\left[\frac{y_{n}}{2}\right]-\left[\frac{y_{n}}{4}\right]+1 \\
& =\tau_{\lambda}^{2}(n)+1
\end{aligned}
$$


Thus (1) holds.

(2) Since $u=v \geq 2$, it follows from Lemma 4.1 that $c_{1,2}=1$. Set

$$
\bar{\Omega}=\Omega-\{(0,0),(1,2)\}
$$

and

$$
\bar{\Gamma}_{n}(y)=\left\{\sigma \in \Gamma_{n} \mid\left[\sigma(1,2,1) / 2^{u-1}\right]=y,\left[\sigma(0,0,1) / 2^{u+1}\right]=y_{n}-y\right\}
$$

for each integer $y$ with $0 \leq y \leq y_{n}$. Then by Proposition 5.1, Lemma 5.1, and (3) of Lemma 6.1,

$$
h_{n}(P) \equiv \sum_{y=0}^{y_{n}} \sum_{\sigma \in \bar{\Gamma}_{n}(y)} h_{n}^{\sigma}(P) \quad \bmod 2^{\tau_{\lambda}^{2}(n)+1} .
$$

Furthermore, if $\sigma \in \bar{\Gamma}_{n}(y)$ with $y \geq 1$ and $y \neq 2$, then $\kappa_{1,2}(\sigma(1,2,1)) \geq 1$, and hence $\rho_{u+1}^{\sigma}(n) \geq 1$ by Lemma 5.2 and (2) of Lemma 5.3. Now, Lemma 5.1 yields

$$
h_{n}(P) \equiv \sum_{\sigma \in \bar{\Gamma}_{n}(0)} h_{n}^{\sigma}(P)+\sum_{\sigma \in \bar{\Gamma}_{n}(2)} h_{n}^{\sigma}(P) \bmod 2^{\tau_{\lambda}^{2}(n)+1} .
$$

Since $c_{0,0}=1$ and $c_{1,2}=1$, it follows that for each $\sigma \in \Gamma_{n}$,

$$
h_{n}^{\sigma}(P)=n ! \frac{h_{n_{0}^{\sigma}}\left(C_{2^{u}} \times C_{2^{u}}\right)}{n_{0}^{\sigma} !} \cdot \frac{h_{n_{1,2}^{\sigma}}\left(C_{2^{u-1}} \times C_{2^{u-2}}\right)}{n_{1,2}^{\sigma} !} \prod_{(l, m) \in \bar{\Omega}} \prod_{i=1}^{c_{l, m}} \frac{g_{l, m}(\sigma(l, m, i))}{\sigma(l, m, i) !}
$$

where $n_{0}^{\sigma}=\sigma(0,0,1)$ and $n_{1,2}^{\sigma}=\sigma(1,2,1)$. Here, if $\sigma \in \bar{\Gamma}_{n}(y)$, then (1) yields $h_{n_{0}^{\sigma}}\left(C_{2^{u}} \times C_{2^{u}}\right) \equiv \frac{n_{0}^{\sigma} ! h_{y_{n}-y}\left(C_{2}\right)}{2^{y_{n}-y}\left(y_{n}-y\right) !} \cdot \frac{h_{n_{0}^{\sigma}-2^{u+1}\left(y_{n}-y\right)}\left(C_{2^{u}} \times C_{2^{u}}\right)}{\left(n_{0}^{\sigma}-2^{u+1}\left(y_{n}-y\right)\right) !} \bmod 2^{\tau_{\lambda}^{2}\left(n_{0}^{\sigma}\right)+1}$.

Also, if $\sigma \in \bar{\Gamma}_{n}(2)$, then $\left[n_{1,2}^{\sigma} / 2^{u}\right]=1$, and, by Theorem 2.1,

$$
h_{n_{1,2}^{\sigma}}\left(C_{2^{u-1}} \times C_{2^{u-2}}\right) \equiv \frac{n_{1,2}^{\sigma} !}{2^{2}} \cdot \frac{h_{n_{1,2}^{\sigma}-2^{u}}\left(C_{2^{u-1}} \times C_{2^{u-2}}\right)}{\left(n_{1,2}^{\sigma}-2^{u}\right) !} \quad \bmod 2^{f_{2}^{u-1}\left(n_{1,2}^{\sigma}\right)}
$$

Now set

$$
h_{n}^{(0)}(P)=\frac{n !}{2^{y_{n}} y_{n} !\left(n-2^{u+1} y_{n}\right) !} h_{y_{n}}\left(C_{2}\right) h_{n-2^{u+1} y_{n}}(P)
$$


and

$$
h_{n}^{(2)}(P)= \begin{cases}\frac{n !}{2^{y_{n}}\left(y_{n}-2\right) !\left(n-2^{u+1} y_{n}\right) !} h_{y_{n}-2}\left(C_{2}\right) h_{n-2^{u+1} y_{n}}(P) & \text { if } \quad y_{n} \geq 2, \\ 0 & \text { otherwise }\end{cases}
$$

Then by an argument similar to the proof of (2) of Theorem 7.1,

$$
\sum_{\sigma \in \bar{\Gamma}_{n}(0)} h_{n}^{\sigma}(P) \equiv h_{n}^{(0)}(P) \quad \bmod 2^{\tau_{\lambda}^{2}(n)+1}
$$

and

$$
\sum_{\sigma \in \bar{\Gamma}_{n}(2)} h_{n}^{\sigma}(P) \equiv h_{n}^{(2)}(P) \quad \bmod 2^{\tau_{\lambda}^{2}(n)+1}
$$

Hence $(\mathrm{N})$ yields

$$
h_{n}(P) \equiv h_{n}^{(0)}(P)+h_{n}^{(2)}(P) \quad \bmod 2^{\tau_{\lambda}^{2}(n)+1} .
$$

We can now show the statements (i), (ii), and (iii) of (2) as follows.

If $y_{n} \geq 2$ and if either $y_{n} \equiv 0 \bmod 4$ or $y_{n} \equiv 1 \bmod 4$, then by $(\mathrm{M})$ and Theorem 5.2,

$$
\begin{aligned}
\operatorname{ord}_{2}\left(h_{n}^{(2)}(P)\right) & \geq \operatorname{ord}_{2}(n !)-y_{n}-\operatorname{ord}_{2}\left(\left(y_{n}-2\right) !\right)+\operatorname{ord}_{2}\left(h_{y_{n}-2}\left(C_{2}\right)\right) \\
& \geq \tau_{\lambda}^{2}(n)+\left[\frac{y_{n}}{4}\right]-\left[\frac{y_{n}-2}{4}\right] \\
& =\tau_{\lambda}^{2}(n)+1
\end{aligned}
$$

Thus (i) follows from $(\mathrm{O})$.

Next, if $y_{n}=2$, then $h_{y_{n}}\left(C_{2}\right)=2$, and hence

$$
h_{n}(P) \equiv \frac{n !}{2} \cdot \frac{h_{n-2^{u+2}}(P)}{\left(n-2^{u+2}\right) !} \equiv 0 \quad \bmod 2^{\tau_{\lambda}^{2}(n)+1}
$$

by (O) and Theorem 5.2. Thus (ii) follows from (2) of Theorem 7.1.

Finally, if $y_{n} \equiv 3 \bmod 4$, then

$$
\operatorname{ord}_{2}\left(h_{n}^{(0)}(P)\right) \geq \tau_{\lambda}^{2}(n)+1
$$

by $(\mathrm{M})$ and Theorem 5.2. Hence (iii) follows from (O). 


\section{Abelian groups}

We generalize Theorems 5.2, 6.1, 7.1, and 7.2, and establish Theorem 1.1 at the end of this section.

Theorem 8.1 Suppose that A possesses a Sylow p-subgroup of type $\lambda$. Let $y_{n}=\left[n / p^{u+1}\right]$ and $z_{n}=\left[n / 2^{u+3}\right]$ for each nonnegative integer $n$. Put $\delta=1$ if either $p>2$ or $s=u$, and is 0 otherwise.

(1) We get $\operatorname{ord}_{p}\left(h_{n}(A)\right) \geq \tau_{\lambda}^{p}(n)$.

(2) Excepting the case where $p=2$ and $2 u=s \geq 2$, if either $u=\lambda_{1}$ or $2 u=s$, then

$$
h_{n}(A) \equiv \frac{(-1)^{y_{n}} n !}{p^{(2 u-s+1) y_{n}} y_{n} !\left(n-p^{u+1} y_{n}\right) !} h_{n-p^{u+1} y_{n}}(A) \quad \bmod p^{\tau_{\lambda}^{p}(n)+2 u-s+\delta} .
$$

(3) If $u>\lambda_{1}$ and if $2 u=s+1$, then

$$
h_{n}(A) \equiv \frac{(-1-p)^{y_{n}} n !}{p^{2 y_{n}} y_{n} !\left(n-p^{u+1} y_{n}\right) !} h_{n-p^{u+1} y_{n}}(A) \quad \bmod p^{\tau_{\lambda}^{p}(n)+1+\delta} .
$$

(4) Assume that $p=2$ and that $2 u=s \geq 2$.

(i) If $\lambda_{3}=0$ and $u \geq 2$, then

$$
h_{n}(A) \equiv \frac{n !}{2^{6 z_{n}} z_{n} !\left(n-2^{u+3} z_{n}\right) !} h_{n-2^{u+3} z_{n}}(A) \bmod 2^{\tau_{\lambda}^{2}(n)+2} .
$$

(ii) If either $\lambda_{3} \geq 1$ or $u=1$, then

$$
h_{n}(A) \equiv \frac{(-1)^{z_{n}} n !}{2^{6 z_{n}} z_{n} !\left(n-2^{u+3} z_{n}\right) !} h_{n-2^{u+3} z_{n}}(A) \quad \bmod 2^{\tau_{\lambda}^{2}(n)+2} .
$$

(5) Assume that $p=2$ and that $2 u=s \geq 2$.

(i) If $\lambda_{3}=0$, then

$$
h_{n}(A) \equiv \frac{n !}{2^{y_{n}} y_{n} !\left(n-2^{u+1} y_{n}\right) !} h_{y_{n}}\left(C_{2}\right) h_{n-2^{u+1} y_{n}}(A) \quad \bmod 2^{\tau_{\lambda}^{2}(n)+1} .
$$

In particular, if $y_{n} \equiv 3 \bmod 4$, then $h_{n}(A) \equiv 0 \bmod 2^{\tau_{\lambda}^{2}(n)+1}$.

(ii) If $\lambda_{3} \geq 1$ and if either $y_{n} \equiv 0 \bmod 4$ or $y_{n} \equiv 1 \bmod 4$, then

$$
h_{n}(A) \equiv \frac{n !}{2^{y_{n}} y_{n} !\left(n-2^{u+1} y_{n}\right) !} h_{y_{n}}\left(C_{2}\right) h_{n-2^{u+1} y_{n}}(A) \quad \bmod 2^{\tau_{\lambda}^{2}(n)+1} .
$$


(iii) If $\lambda_{3} \geq 1$ and if $y_{n} \equiv 2 \bmod 4$, then $h_{n}(A) \equiv 0 \bmod 2^{\tau_{\lambda}^{2}(n)+1}$.

(iv) If $\lambda_{3} \geq 1$ and if $y_{n} \equiv 3 \bmod 4$, then

$$
\begin{array}{r}
h_{n}(A) \equiv \frac{n !}{2^{y_{n}}\left(y_{n}-2\right) !\left(n-2^{u+1} y_{n}\right) !} h_{y_{n}-2}\left(C_{2}\right) h_{n-2^{u+1} y_{n}}(A) \\
\bmod 2^{\tau_{\lambda}^{2}(n)+1} .
\end{array}
$$

Remark 8.1 The first assertion of $[9$, Theorem 5.1(3)] with $l \geq 2$ is wrong.

Although we can get Theorem 8.1 as an analogy with [9, Theorem 5.1], we give an orderly proof of it. Let $P$ be a finite abelian $p$-group of type $\lambda$. For a positive integer $a$, we define

$$
E_{P}(a ; X):=\exp \left(\sum_{k=0}^{s} \frac{\alpha_{\lambda}(s-k ; p)}{a p^{k}} X^{a p^{k}}\right)
$$

and denote by $h_{a n}(a ; P), n=0,1,2, \ldots$, the rational numbers such that

$$
E_{P}(a ; X)=\sum_{n=0}^{\infty} \frac{h_{a n}(a ; P)}{(a n) !} X^{a n} .
$$

Especially, $h_{n}(P)=h_{n}(1 ; P)$. This power series is introduced in [9, Section 5]. Under the notation above, we get the following.

Proposition 8.1 Suppose that $a_{0}=1$ and that $a_{1}, \ldots, a_{l}$ are integers such that $a_{i}>1$ and $\operatorname{gcd}\left(a_{i}, p\right)=1$ for all $i$. Let $m, n_{0}, n_{1}, \ldots, n_{l}$ be nonnegative integers with $m \geq a_{0} n_{0}+a_{1} n_{1}+\cdots+a_{l} n_{l}$. Put

$$
H\left(m: a_{1}, \ldots, a_{l} ; n_{0}, \ldots, n_{l}\right)=\frac{m !}{h_{n_{0}}(P)} \prod_{i=0}^{l} \frac{h_{a_{i} n_{i}}\left(a_{i} ; P\right)}{\left(a_{i} n_{i}\right) !} .
$$

Then $\operatorname{ord}_{p}\left(H\left(m: a_{1}, \ldots, a_{l} ; n_{0}, \ldots, n_{l}\right)\right) \geq \tau_{\lambda}^{p}(m)-\tau_{\lambda}^{p}\left(n_{0}\right)$, and the following statements hold.

(1) If $\left[n_{0} / p^{u+1}\right]<\left[m / p^{u+1}\right]$, then

$$
\operatorname{ord}_{p}\left(H\left(m: a_{1}, \ldots, a_{l} ; n_{0}, \ldots, n_{l}\right)\right) \geq \tau_{\lambda}^{p}(m)+2 u-s+1-\tau_{\lambda}^{p}\left(n_{0}\right) .
$$

(2) Assume that $p=2$ and that $2 u=s \geq 2$. If $\left[n_{0} / 2^{u+3}\right]<\left[m / 2^{u+3}\right]$, then

$$
\operatorname{ord}_{2}\left(H\left(m: a_{1}, \ldots, a_{l} ; n_{0}, \ldots, n_{l}\right)\right) \geq \tau_{\lambda}^{2}(m)+2-\tau_{\lambda}^{2}\left(n_{0}\right) .
$$


Proposition 8.1 is a result of the following three lemmas. Under the hypothesis and notation of Proposition 8.1, we show a technical lemma:

Lemma 8.1 Suppose that $\operatorname{ord}_{p}\left(h_{a_{i} n_{i}}\left(a_{i} ; P\right)\right) \geq \tau_{\lambda}^{p}\left(a_{i} n_{i}\right)$ for all $i$ with $i \geq 1$. Then $\operatorname{ord}_{p}\left(H\left(m: a_{1}, \ldots, a_{l} ; n_{0}, \ldots, n_{l}\right)\right) \geq \tau_{\lambda}^{p}(m)-\tau_{\lambda}^{p}\left(n_{0}\right)$, and the following statements hold.

(1) Suppose that $\operatorname{ord}_{p}\left(h_{a_{i} n_{i}}\left(a_{i} ; P\right)\right) \geq \tau_{\lambda}^{p}\left(a_{i} n_{i}\right)+2 u-s+1$, provided $i \geq 1$ and $a_{i} n_{i} \geq p^{u+1}$. Then the assertion (1) of Proposition 8.1 holds.

(2) Assume that $p=2$ and that $2 u=s \geq 2$. Moreover, suppose that $\operatorname{ord}_{2}\left(h_{a_{i} n_{i}}\left(a_{i} ; P\right)\right) \geq \tau_{\lambda}^{2}\left(a_{i} n_{i}\right)+2$, provided $i \geq 1$ and $a_{i} n_{i} \geq 2^{u+3}$. Then the assertion (2) of Proposition 8.1 holds.

Proof. Unless $p=2$ and $2 u=s \geq 2$,

$$
\begin{aligned}
& \operatorname{ord}_{p}(\left.H\left(m: a_{1}, \ldots, a_{l} ; n_{0}, \ldots, n_{l}\right)\right)+\operatorname{ord}_{p}\left(h_{n_{0}}(P)\right) \\
&= \sum_{j=1}^{\infty}\left[\frac{m}{p^{j}}\right]+\sum_{i=0}^{l}\left(\operatorname{ord}_{p}\left(h_{a_{i} n_{i}}\left(a_{i} ; P\right)\right)-\sum_{j=1}^{\infty}\left[\frac{a_{i} n_{i}}{p^{j}}\right]\right) \\
& \geq \tau_{\lambda}^{p}(m)+(2 u-s+1)\left[\frac{m}{p^{u+1}}\right]+\sum_{i=0}^{l}\left(\operatorname{ord}_{p}\left(h_{a_{i} n_{i}}\left(a_{i} ; P\right)\right)-\sum_{j=1}^{u+1}\left[\frac{a_{i} n_{i}}{p^{j}}\right]\right) \\
&=\tau_{\lambda}^{p}(m)+(2 u-s+1)\left(\left[\frac{m}{p^{u+1}}\right]-\sum_{i=0}^{l}\left[\frac{a_{i} n_{i}}{p^{u+1}}\right]\right) \\
&+\sum_{i=0}^{l}\left(\operatorname{ord}_{p}\left(h_{a_{i} n_{i}}\left(a_{i} ; P\right)\right)-\tau_{\lambda}^{p}\left(a_{i} n_{i}\right)\right) .
\end{aligned}
$$

Likewise, if $p=2$ and $2 u=s \geq 2$, then

$$
\begin{aligned}
\operatorname{ord}_{2}\left(H\left(m: a_{1}, \ldots, a_{l} ; n_{0}, \ldots, n_{l}\right)\right)+\operatorname{ord}_{p}\left(h_{n_{0}}(P)\right) \\
\geq \tau_{\lambda}^{2}(m)+\left[\frac{m}{2^{u+1}}\right]+2\left[\frac{m}{2^{u+3}}\right]+\sum_{i=0}^{l}\left(\operatorname{ord}_{2}\left(h_{a_{i} n_{i}}\left(a_{i} ; P\right)\right)-\sum_{j=1}^{u+3}\left[\frac{a_{i} n_{i}}{2^{j}}\right]\right) \\
=\tau_{\lambda}^{2}(m)+\left(\left[\frac{m}{2^{u+1}}\right]-\sum_{i=0}^{l}\left[\frac{a_{i} n_{i}}{2^{u+1}}\right]\right)+2\left(\left[\frac{m}{2^{u+3}}\right]-\sum_{i=0}^{l}\left[\frac{a_{i} n_{i}}{2^{u+3}}\right]\right) \\
+\sum_{i=0}^{l}\left(\operatorname{ord}_{2}\left(h_{a_{i} n_{i}}\left(a_{i} ; P\right)\right)-\tau_{\lambda}^{2}\left(a_{i} n_{i}\right)\right) .
\end{aligned}
$$

The lemma follows from these inequalities and Theorem 5.2.

The following lemma is a consequence of Theorem 5.2 and Lemma 8.1. 
Lemma 8.2 Let a be an integer greater than 1 . Then $\operatorname{ord}_{p}\left((\right.$ an $\left.) ! h_{n}(P) / n !\right) \geq$ $\tau_{\lambda}^{p}($ an $)$, and the following statements hold.

(1) We have $\operatorname{ord}_{p}\left((\right.$ an $\left.) ! h_{n}(P) / n !\right) \geq \tau_{\lambda}^{p}($ an $)+2 u-s+1$ provided an $\geq p^{u+1}$.

(2) Assume that $p=2$ and that $2 u=s \geq 2$. Then $\operatorname{ord}_{2}\left((a n) ! h_{n}(P) / n !\right) \geq$ $\tau_{\lambda}^{2}($ an $)+2$ provided an $\geq 2^{u+3}$.

Proof. If $a n \geq p^{u+1}$, then $\left[n / p^{u+1}\right]<\left[a n / p^{u+1}\right]$, because $a>1$. Also, if an $\geq 2^{u+3}$, then $\left[n / 2^{u+3}\right]<\left[a n / 2^{u+3}\right]$. Now, Theorem 5.2 and Lemma 8.1 with $m=a n, n_{0}=n$, and $l=0$ yield the lemma. This completes the proof.

Using Lemmas 8.1 and 8.2, we obtain the following generalization of $[9$, Lemma 5.1], which, together with Lemma 8.1, yields Proposition 8.1.

Lemma 8.3 Let $a$ be an integer such that $a>1$ and $\operatorname{gcd}(a, p)=1$. Then $\operatorname{ord}_{p}\left(h_{a n}(a ; P)\right) \geq \tau_{\lambda}^{p}($ an $)$, and the following statements hold.

(1) We have $\operatorname{ord}_{p}\left(h_{a n}(a ; P)\right) \geq \tau_{\lambda}^{p}($ an $)+(2 u-s+1)$ provided an $\geq p^{u+1}$.

(2) Assume that $p=2$ and that $2 u=s \geq 2$. Then $\operatorname{ord}_{2}\left(h_{a n}(a ; P)\right) \geq$ $\tau_{\lambda}^{2}($ an $)+2$ provided an $\geq 2^{u+3}$.

Proof. We show the lemma by induction on $n$. If $n=0$, then the assertions clearly hold. Assume that $n>0$. By the definition, $E_{P}(a ; X)^{a}=E_{P}\left(X^{a}\right)$. Comparing the coefficients of $X^{a n}$ on both sides, we have

$$
a h_{a n}(a ; P)+\sum_{\substack{n_{1}+n_{2}+\cdots+n_{a}=n \\ n_{i}<n}}(a n) ! \prod_{i=1}^{a} \frac{h_{a n_{i}}(a ; P)}{\left(a n_{i}\right) !}=(a n) ! \frac{h_{n}(P)}{n !}
$$

where the summation runs over all sequences $n_{1}, n_{2}, \ldots, n_{a}$ of nonnegative integers such that $n_{1}+n_{2}+\cdots+n_{a}=n$ and $n_{i}<n$ for all $i$. Now, since $\operatorname{gcd}(a, p)=1$, the inductive assumption and Lemma 8.1 with $m=a n, n_{0}=0$, $l=a$, and $a_{1}=\cdots=a_{l}=a$, together with Lemma 8.2, yield the assertions. This completes the proof.

Proof of Proposition 8.1. The statements follow from Lemmas 8.1 and 8.3.

We can now prove Theorem 8.1. 
Proof of Theorem 8.1. Let $P$ be a Sylow $p$-subgroup of $A$. Then there exist integers $a_{1}, a_{2}, \ldots, a_{l}$ greater than 1 such that

$$
E_{A}(X)=E_{P}(X) \prod_{i=1}^{l} E_{P}\left(a_{i} ; X\right)
$$

Comparing the coefficients of $X^{n}$ on both sides, we have

$$
h_{n}(A)=\sum_{n_{0}=0}^{n} h_{n_{0}}(P) \sum_{a_{1} n_{1}+\cdots+a_{l} n_{l}=n-n_{0}} H\left(n: a_{1}, \ldots, a_{l} ; n_{0}, \ldots, n_{l}\right),
$$

where the summation $\sum_{a_{1} n_{1}+\cdots+a_{l} n_{l}=n-n_{0}}$ runs over all sequences $n_{1}, \ldots, n_{l}$ of nonnegative integers such that $a_{1} n_{1}+\cdots+a_{l} n_{l}=n-n_{0}$. (For the notation, see Proposition 8.1.) Hence (1) follows from Theorem 5.2 and Proposition 8.1. Moreover, in the equation above, if $n_{0}<p^{u+1} y_{n}$, then

$$
\operatorname{ord}_{p}\left(H\left(n: a_{1}, \ldots, a_{l} ; n_{0}, \ldots, n_{l}\right)\right) \geq \tau_{\lambda}^{p}(n)+2 u-s+1-\tau_{\lambda}^{p}\left(n_{0}\right)
$$

by (1) of Proposition 8.1. Now we have

$$
\begin{aligned}
h_{n}(A) \equiv n ! \sum_{n_{0} \geq p^{u+1} y_{n}} \frac{h_{n_{0}}(P)}{n_{0} !} \sum_{a_{1} n_{1}+\cdots+a_{l} n_{l}=n-n_{0}} \prod_{i=1}^{l} \frac{h_{a_{i} n_{i}}\left(a_{i} ; P\right)}{\left(a_{i} n_{i}\right) !} \\
\bmod p^{\tau_{\lambda}^{p}(n)+2 u-s+1} .
\end{aligned}
$$

Excepting the case where $p=2$ and $2 u=s \geq 2$, if either $u=\lambda_{1}$ or $2 u=s$, then by (1) of Theorem 6.1 and Proposition 8.1,

$$
\begin{aligned}
h_{n}(A) \equiv n ! \frac{(-1)^{y_{n}}}{p^{(2 u-s+1) y_{n}} y_{n} !} \sum_{n_{0} \geq p^{u+1} y_{n}} \frac{h_{n_{0}-p^{u+1} y_{n}}(P)}{\left(n_{0}-p^{u+1} y_{n}\right) !} \\
\times \sum_{a_{1} n_{1}+\cdots+a_{l} n_{l}=n-n_{0}} \prod_{i=1}^{l} \frac{h_{a_{i} n_{i}}\left(a_{i} ; P\right)}{\left(a_{i} n_{i}\right) !} \\
\equiv \frac{(-1)^{y_{n}} n !}{p^{(2 u-s+1) y_{n}} y_{n} !\left(n-p^{u+1} y_{n}\right) !} h_{n-p^{u+1} y_{n}}(A) \bmod p^{\tau_{\lambda}^{p}(n)+2 u-s+\delta} .
\end{aligned}
$$

Hence (2) holds. Likewise, (3) and (5) follow from Theorems 6.1(2) and 7.2 and Proposition 8.1, and (4) follows from Theorem 7.1 and Proposition 8.1. This completes the proof.

The proof of the following corollary to Theorem 8.1 is completely analogous to that of [9, Corollary 3.1]. 
Corollary 8.1 Under the hypothesis of Theorem 8.1, the following statements hold.

(1) Assume that either $p>2$ or $2 u>s$.

(i) If $\operatorname{ord}_{p}\left(h_{n-p^{u+1} y_{n}}(A)\right)<\tau_{\lambda}^{p}\left(n-p^{u+1} y_{n}\right)+2 u-s+\delta$, then

$$
\operatorname{ord}_{p}\left(h_{n}(A)\right)=\operatorname{ord}_{p}\left(h_{n-p^{u+1} y_{n}}(A)\right)+\tau_{\lambda}^{p}\left(p^{u+1} y_{n}\right) .
$$

(ii) If $\operatorname{ord}_{p}\left(h_{n-p^{u+1} y_{n}}(A)\right) \geq \tau_{\lambda}^{p}\left(n-p^{u+1} y_{n}\right)+2 u-s+\delta$, then

$$
\operatorname{ord}_{p}\left(h_{n}(A)\right) \geq \tau_{\lambda}^{p}(n)+2 u-s+\delta .
$$

(2) Assume that $p=2$ and that $2 u=s \geq 2$.

(i) If $\operatorname{ord}_{2}\left(h_{n-2^{u+3} z_{n}}(A)\right)<\tau_{\lambda}^{2}\left(n-2^{u+3} z_{n}\right)+2$, then

$$
\operatorname{ord}_{2}\left(h_{n}(A)\right)=\operatorname{ord}_{2}\left(h_{n-2^{u+3} z_{n}}(A)\right)+\tau_{\lambda}^{2}\left(2^{u+3} z_{n}\right) .
$$

(ii) If $\operatorname{ord}_{2}\left(h_{n-2^{u+3} z_{n}}(A)\right) \geq \tau_{\lambda}^{2}\left(n-2^{u+3} z_{n}\right)+2$, then

$$
\operatorname{ord}_{2}\left(h_{n}(A)\right) \geq \tau_{\lambda}^{2}(n)+2 .
$$

The assertion (1) of the following corollary is a part of [9, Theorem 1.4].

Corollary 8.2 Assume that $p=2$ and that $2 u=s \geq 2$.

(1) If $\lambda_{3}=0$, then $\operatorname{ord}_{2}\left(h_{n}(A)\right)=\tau_{\lambda}^{2}(n)$ for each nonnegative integer $n$ such that $n \equiv 0,2^{u+1}$, or $2^{u+2} \bmod 2^{u+3}$.

(2) If $\lambda_{3} \geq 1$, then $\operatorname{ord}_{2}\left(h_{n}(A)\right)=\tau_{\lambda}^{2}(n)$ for each nonnegative integer $n$ such that $n \equiv 0,2^{u+1}$, or $2^{u+1}+2^{u+2} \bmod 2^{u+3}$.

Proof. As mentioned earlier, for any positive integer $y$,

$$
\operatorname{ord}_{2}\left(h_{y}\left(C_{2}\right)\right)=\left[\frac{y}{2}\right]-\left[\frac{y}{4}\right]
$$

if $y \not \equiv 3 \bmod 4$ (see $(\mathrm{M}))$. Hence (5) of Theorem 8.1 yields the corollary.

Theorem 1.1 is a consequence of Theorem 8.1 and Corollaries 8.1 and 8.2.

Proof of Theorem 1.1. The assertion (1) follows from (1) of Theorem 8.1 and (1)(i) of Corollary 8.1. The assertion (2) follows from Corollary 8.2. 


\section{$9 \quad$ The proof of Corollary 1.1}

We conclude this paper with the proof of Corollary 1.1.

Lemma 9.1 Suppose that A possesses a Sylow p-subgroup of type $\lambda$. Set

$$
a=\lim _{n \rightarrow \infty} \inf \frac{\operatorname{ord}_{p}\left(h_{n}(A)\right)-\operatorname{ord}_{p}(n !)}{n} .
$$

Then

$$
a= \begin{cases}-\frac{7}{2^{u+3}} & \text { if } p=2 \text { and if } 2 u=s \geq 2, \\ -\left(\frac{1}{p-1}+2 u-s+1\right) \frac{1}{p^{u+1}} & \text { otherwise. }\end{cases}
$$

Proof. Suppose that $p=2$ and $2 u=s \geq 2$. For each positive integer $n$, set

$$
a_{n}=\frac{\operatorname{ord}_{2}\left(h_{n}(A)\right)-\operatorname{ord}_{2}(n !)}{n}
$$

and $z_{n}=\left[n / 2^{u+3}\right]$. Then (2) of Corollary 8.1, together with (1) of Theorem 8.1, yields

$$
\begin{aligned}
a_{n} & \geq-\frac{1}{n}\left(\left[\frac{n}{2^{u+1}}\right]+2 z_{n}+\sum_{j=1}^{\infty}\left[\frac{z_{n}}{2^{j}}\right]\right) \\
& \geq-\frac{1}{2^{u+3} z_{n}+\left(n-2^{u+3} z_{n}\right)}\left(\left[\frac{n-2^{u+3} z_{n}}{2^{u+1}}\right]+\operatorname{ord}_{2}\left(2^{6 z_{n}} z_{n} !\right)\right) \\
& \geq-\frac{\operatorname{ord}_{2}\left(2^{6 z_{n}} z_{n} !\right)}{2^{u+3} z_{n}} \\
& =a_{2^{u+3} z_{n}} .
\end{aligned}
$$

By [10, Chapter IV], we have

$$
\begin{aligned}
\lim _{n \rightarrow \infty} a_{2^{u+3} z_{n}} & =-\frac{6}{2^{u+3}}-\frac{1}{2^{u+3}} \lim _{n \rightarrow \infty} \frac{\operatorname{ord}_{2}\left(z_{n} !\right)}{z_{n}} \\
& =-\frac{7}{2^{u+3}} .
\end{aligned}
$$

We now define a sequence $\left\{\beta_{n}\right\}_{n=1}^{\infty}$ by

$$
\beta_{n}= \begin{cases}a_{n} & \text { if } n=2^{u+3} z_{n}, \\ a_{2^{u+3}\left(z_{n}+1\right)} & \text { otherwise. }\end{cases}
$$


Set $l_{n}=\inf \left\{a_{i} \mid i \geq n\right\}$ and $m_{n}=\inf \left\{\beta_{i} \mid i \geq n\right\}$. Then $\left\{l_{n}\right\}_{n=1}^{\infty}$ and $\left\{m_{n}\right\}_{n=1}^{\infty}$ are monotone sequences satisfying

$$
l_{1} \leq l_{2} \leq \cdots \leq l_{n} \leq m_{n} \leq m_{n+1} \leq \cdots .
$$

Moreover,

$$
\lim _{n \rightarrow \infty} m_{n}=\lim _{n \rightarrow \infty} a_{2^{u+3} z_{n}}=-\frac{7}{2^{u+3}},
$$

and thereby, $\left\{l_{n}\right\}_{n=1}^{\infty}$ converges. Since $l_{2^{u+3} z}=m_{2^{u+3} z}$ for any positive integer $z$, it follows that

$$
a=\lim _{n \rightarrow \infty} l_{n}=\lim _{n \rightarrow \infty} m_{2^{u+3} z_{n}}=\lim _{n \rightarrow \infty} m_{n}=-\frac{7}{2^{u+3}} .
$$

Unless $p=2$ and $2 u=s \geq 2$, a similar argument to the preceding one, together with (1) of Corollary 8.1 , enables us to obtain

$$
a=-\left(\frac{1}{p-1}+2 u-s+1\right) \frac{1}{p^{u+1}}
$$

Thus the lemma follows.

Proof of Corollary 1.1. By [10, Chapter IV] and Lemma 9.1, the radius of convergence is $p^{a}$, where

$$
a= \begin{cases}-\frac{7}{2^{u+3}} & \text { if } p=2 \text { and if } 2 u=s \geq 2 \\ -\left(\frac{1}{p-1}+2 u-s+1\right) \frac{1}{p^{u+1}} & \text { otherwise }\end{cases}
$$

(see also [9]). Suppose that $|x|_{p}=p^{a}$ (see [10]). If $p=2$ and if $2 u=s \geq 2$, then by (2) of Theorem 1.1,

$$
\operatorname{ord}_{2}\left(\frac{h_{2^{u+3+k}}(A)}{2^{u+3+k} !} x^{2^{u+3+k}}\right)=-2^{u+3+k} a-7 \cdot 2^{k}+1=1
$$

for any nonnegative integer $k$. Unless $p=2$ and $2 u=s \geq 2$, (1) of Theorem 1.1 , yields

$$
\operatorname{ord}_{p}\left(\frac{h_{p^{u+1+k}}(A)}{p^{u+1+k} !} x^{p^{u+1+k}}\right)=-p^{u+1+k} a-(2 u-s+1) p^{k}-\frac{p^{k}-1}{p-1}=\frac{1}{p-1}
$$

for any nonnegative integer $k$. Hence the $p$-adic power series $E_{A}(X)$ converges only in the open disc of radius $p^{a}$. This completes the proof. 
Remark 9.1 The radius of convergence of the $p$-adic power series $E_{C_{p}}(X)$ is given in [14, p. 389, Proposition].

\section{ACKNOWLEDGMENT}

The author would like to thank Hidenori Katsurada and Tomoyuki Yoshida for their helpful advice.

\section{References}

[1] L. M. Butler, A unimodality result in the enumeration of subgroups of a finite abelian group, Proc. Amer. Math. Soc. 101 (1987), 771-775.

[2] N. Chigira, The solutions of $x^{d}=1$ in finite groups, J. Algebra 180 (1996), 653-661.

[3] S. Chowla, I. N. Herstein, and W. K. Moore, On recursions connected with symmetric groups I, Canad. J. Math. 3 (1951), 328-334.

[4] A. W. M. Dress and T. Yoshida, On p-divisibility of the Frobenius numbers of symmetric groups, 1991, unpublished note.

[5] B. Dwork, A note on the p-adic gamma function, Groupe d'étude d'Analyse ultramétrique, 9e année, 1981/82, fasc. 3, n J5, 10 pp.

[6] M. Grady and M. Newman, Residue periodicity in subgroup counting functions; in :"The Rademacher Legacy to Mathematics," Contemp. Math. 166 (1994), 265-273.

[7] H. Ishihara, H. Ochiai, Y. Takegahara, and T. Yoshida, p-divisibility of the number of solutions of $x^{p}=1$ in a symmetric group, Ann. Comb. 5 (2001), 197-210.

[8] G. D. James and A. Kerber, The Representation Theory of the Symmetric Group, Encyclopedia of mathematics and its applications, Vol. 16, Addison-Wesley, Reading, MA, 1981.

[9] H. Katsurada, Y. Takegahara, and T. Yoshida, The number of homomorphisms from a finite abelian group to a symmetric group, Comm. Algebra 28 (2000), 2271-2290.

[10] N. Koblitz, p-adic Numbers, p-adic Analysis, and Zeta-Functions, 2nd ed., Springer-Verlag, New York, 1984.

[11] M. Murai, On the number of $p$-subgroups of a finite group, J. Math. Kyoto Univ. 42 (2002), 161-174. 
[12] H. Ochiai, A $p$-adic property of the Taylor series of $\exp \left(x+x^{p} / p\right)$, Hokkaido Math. J. 28 (1999), 71-85.

[13] S. Okada, Wreath products by the symmetric groups and product posets of Young's lattices, J. Combin. Theory Ser. A 55 (1990), 14-32.

[14] A. M. Robert, A Course in p-adic Analysis, Springer-Verlag, New York, 2000 .

[15] T. Stehling, On computing the number of subgroups of a finite abelian group, Combinatorica 12 (1992), 475-479.

[16] Y. Takegahara, On Butler's unimodality result, Combinatorica, 18 (1998), 437-439.

[17] Y. Takegahara, On the Frobenius numbers of symmetric groups, J. Algebra 221 (1999), 551-561.

[18] Y. Takegahara, On Wohlfahrt formula for finite abelian groups; Formal power series and algebraic combinatorics (Moscow, 2000), 754-764, Springer-Verlag, Berlin, 2000.

[19] Y. Takegahara, On Wohlfahrt series and wreath products, Adv. Math. 209 (2007), 526-546.

[20] K. Wohlfahrt, Über einen Satz von Dey und die Modulgruppe, Arch. Math. (Basel) 29 (1977), 455-457.

[21] T. Yoshida, $|\operatorname{Hom}(A, G)|$, J. Algebra 156 (1993), 125-156. 